\title{
$(m, \lambda)$-Berezin Transform on the Weighted Bergman Spaces over the Polydisk
}

\author{
Ran Li and Yufeng Lu \\ School of Mathematical Sciences, Dalian University of Technology, Dalian 116024, China \\ Correspondence should be addressed to Ran Li; liranmika@163.com
}

Received 10 March 2016; Revised 30 August 2016; Accepted 8 September 2016

Academic Editor: Alberto Fiorenza

Copyright (C) $2016 \mathrm{R}$. Li and Y. Lu. This is an open access article distributed under the Creative Commons Attribution License, which permits unrestricted use, distribution, and reproduction in any medium, provided the original work is properly cited.

We prove that every bounded linear operator on weighted Bergman space over the polydisk can be approximated by Toeplitz operators under some conditions. The main tool here is the so-called $(m, \lambda)$-Berezin transform. In particular, our results generalized the results of K. Nam and D. C. Zheng to the case of operators acting on $\mathscr{A}_{\lambda}^{2}\left(\mathbb{D}^{n}\right)$.

\section{Introduction}

Let $\mathbb{D}$ be the unit disk in $\mathbb{C}$ and $d A_{\mu}(z)=c_{\mu}\left(1-|z|^{2}\right)^{\mu} d A(z)$ be a positive standard weighted probability measure on $\mathbb{D}$, where the weighted parameter fulfills $\mu>-1$ and the normalized constant $c_{\mu}=\mu+1$. For a fixed positive integer $n$, the polydisk $\mathbb{D}^{n}$ is the Cartesian product of $n$ copies of $\mathbb{D}$ and

$$
d A_{\lambda}(z)=d A_{\lambda_{1}}\left(z_{1}\right) \cdots d A_{\lambda_{n}}\left(z_{n}\right)
$$

is the normalized weighted Lebesgue volume measure on the polydisk $\mathbb{D}^{n}$. The Bergman space $\mathscr{A}_{\lambda}^{2}\left(\mathbb{D}^{n}\right)=\mathscr{A}_{\lambda}^{2}\left(\mathbb{D}^{n}, d A_{\lambda}\right)$ is the set of all analytic functions on $\mathbb{D}^{n}$ in $L_{\lambda}^{2}\left(\mathbb{D}^{n}, d A_{\lambda}\right)=$ $L_{\lambda}^{2}\left(\mathbb{D}^{n}\right)$. As is well known $\mathscr{A}_{\lambda}^{2}\left(\mathbb{D}^{n}\right)$ forms a closed subspace of $L_{\lambda}^{2}\left(\mathbb{D}^{n}\right)$ and has the structure of reproducing kernel Hilbert space. We denote by $B_{\lambda}$ the Bergman projection of $L_{\lambda}^{2}\left(\mathbb{D}^{n}\right)$ onto $\mathscr{A}_{\lambda}^{2}\left(\mathbb{D}^{n}\right)$. In case of $\lambda=0, \mathscr{A}_{0}^{2}\left(\mathbb{D}^{n}\right)$ is the unweighted Bergman space denoted by $\mathscr{A}^{2}\left(\mathbb{D}^{n}\right)$. Given an essentially bounded measurable function $a \in L^{\infty}\left(\mathbb{D}^{n}\right)$, we write $T_{a}$ for the Toeplitz operator with the symbol $a$, which acts on $\mathscr{A}_{\lambda}^{2}\left(\mathbb{D}^{n}\right)$ as $T_{a} f=B_{\lambda}(a f)$. That is, the Toeplitz operator is defined as the compression of a multiplication operator on $L_{\lambda}^{2}\left(\mathbb{D}^{n}\right)$ onto the Bergman space. The Toeplitz algebra $\mathfrak{I}\left(L^{\infty}\right)$ is the closed subalgebra of $\mathscr{L}\left(\mathscr{A}_{\lambda}^{2}\right)$ generated by $\left\{T_{a}: a \in\right.$ $\left.L^{\infty}\left(\mathbb{D}^{n}\right)\right\}$, where $\mathscr{L}\left(\mathscr{A}_{\lambda}^{2}\right)$ denotes the algebra of all bounded linear operators on $\mathscr{A}_{\lambda}^{2}\left(\mathbb{D}^{n}\right)$.

Due to their simple structure Toeplitz operators form an important, tractable, and intensively studied subclass in the algebra $\mathscr{L}\left(\mathscr{A}_{\lambda}^{2}\right)$ of all bounded linear operators on $\mathscr{A}_{\lambda}^{2}\left(\mathbb{D}^{n}\right)$. The natural question is whether the Toeplitz algebra is dense in the algebra of all bounded linear operators on the Bergman space. On unweighted Bergman space over the unit disk and even more general domain in $\mathbb{C}$, it is proved in [1] that the Toeplitz algebra is dense in the algebra of all bounded linear operators in the sense of strong operator topology (SOT). In general, it is not true if the SOT is replaced by the norm topology.

Nam and Zheng give a criterion for bounded operators approximated by Toeplitz operators on $\mathscr{A}^{2}\left(\mathbb{D}^{n}\right)$. Since the Berezin transform is a useful tool to study operators on any reproducing kernel Hilbert space, the $m$-Berezin transform for any bounded linear operators acting on $\mathscr{A}^{2}\left(\mathbb{D}^{n}\right)$ was defined in [2]. The operator $S \in \mathscr{L}\left(L_{a}^{2}\right)$ can be approximated in the norm by Toeplitz operators on the unit ball (see [3]) by using the $m$-Berezin transform. In [4], the $(k, \alpha)$ Berezin transform for complex-valued regular measures on the weighted $p$-Bergman space over the unit ball was defined and studied. Using it, they show that every $S \in$ $\mathfrak{I}\left(L^{\infty}\right)$ can be approximated by certain localized operators and introduce a way to connect the behavior of these localized operators with the Berezin transform. The $(m, \lambda)$-Berezin transform for general bounded operators acting on the weighted Bergman space $\mathscr{A}_{\lambda}^{2}\left(\mathbb{B}^{n}\right)$ was defined in [5] and the authors establish various results on norm approximations via the $(m, \lambda)$-Berezin transform and describe conditions under which a bounded linear operator $S$ can be approximated 
in norm by Toeplitz operators whose symbols are bounded functions.

In this paper, we will define and study the $(m, \lambda)$-Berezin transform for general bounded operators acting on the weighted Bergman space $\mathscr{A}_{\lambda}^{2}\left(\mathbb{D}^{n}\right)$ in the third section. The $(m, \lambda)$-Berezin transform of a Toeplitz operator $T_{a}$ acting on $\mathscr{A}_{\lambda}^{2}\left(\mathbb{D}^{n}\right)$ coincides with $(0, \lambda+m)$-Berezin transform for $T_{a}$ considered on the weighted Bergman space $\mathscr{A}_{\lambda+m}^{2}\left(\mathbb{D}^{n}\right)$. We will show that the $(m, \lambda)$-Berezin transforms are commuting with each other. In Section 4, we will establish various results on norm approximation by the $(m, \lambda)$-Berezin transform. More precisely, we describe how to approximate a bounded linear operator $S$ on $\mathscr{A}_{\lambda}^{2}\left(\mathbb{D}^{n}\right)$ in norm by Toeplitz operators whose symbols are bounded functions which are given as the $(m, \lambda)$-Berezin transform of the initial operator $S$ under some conditions. We would like to point out that these results generalize ideas and theorems in [2] to the case of operators acting on $\mathscr{A}_{\lambda}^{2}\left(\mathbb{D}^{n}\right)$.

\section{Preliminaries}

Let $\mathbb{D}^{n}=\left\{z=\left(z_{1}, \ldots, z_{n}\right):\left|z_{i}\right|<1\right.$, for $\left.i=1, \ldots, n\right\}$ be the polydisk in $\mathbb{C}^{n}$ equipped with the standard weighted measure (1), where $\lambda=\left(\lambda_{1}, \ldots, \lambda_{n}\right)$ is fixed and $\lambda_{i}>-1$ for any $i=$ $1, \ldots, n$. For a vector $\lambda=\left(\lambda_{1}, \ldots, \lambda_{n}\right) \in \mathbb{R}^{n}$ and a positive integer $m$ we will employ the notations

$$
\begin{aligned}
\lambda+m & =\left(\lambda_{1}+m, \ldots, \lambda_{n}+m\right), \\
|\lambda+m| & =\sum_{i=1}^{n}\left(\lambda_{i}+m\right)=n m+\sum_{i=1}^{n} \lambda_{i}, \\
{[\lambda+m] } & =\prod_{i=1}^{n}\left(\lambda_{i}+m\right) .
\end{aligned}
$$

In addition, if $\lambda_{i}$ is a positive integer for any $i=1, \ldots, n$ and $m>0, \lambda$ and $\lambda+m$ are multi-index. Let $\mathbb{Z}_{+}:=\{0,1, \ldots\}$ be the set of nonnegative integers. With $\alpha \in \mathbb{Z}_{+}^{n}$, we use the standard notations $z^{\alpha}:=z_{1}^{\alpha_{1}} \cdots z_{n}^{\alpha_{n}}, \alpha !:=\alpha_{1} ! \cdots \alpha_{n}$ ! and $|\alpha|:=$ $\alpha_{1}+\cdots+\alpha_{n}$.

As we all know, for all $\alpha \in \mathbb{Z}_{+}^{n}$ and $\lambda=\left(\lambda_{1}, \ldots, \lambda_{n}\right)$, where $\lambda_{i}>-1$, for $i=1, \ldots, n$, we have

$$
\left\|w^{\alpha}\right\|_{2, \lambda}=\sqrt{[\lambda+1] \prod_{i=1}^{n} \frac{\Gamma\left(\alpha_{i}+1\right) \Gamma\left(\lambda_{i}+1\right)}{\Gamma\left(\alpha_{i}+\lambda_{i}+2\right)}}
$$

and then $\left\{e_{\alpha}=w^{\alpha}\left\|w^{\alpha}\right\|_{2, \lambda}^{-1}: \alpha \in \mathbb{Z}_{+}^{n}\right\}$ is the standard orthonormal basis of $\mathscr{A}_{\lambda}^{2}\left(\mathbb{D}^{n}\right)$. The reproducing kernel in $\mathscr{A}_{\lambda}^{2}\left(\mathbb{D}^{n}\right)$ is given by

$$
K_{z}^{\lambda}(w)=\prod_{i=1}^{n} \frac{1}{\left(1-\overline{z_{i}} w_{i}\right)^{2+\lambda_{i}}}
$$

for $z, w \in \mathbb{D}^{n}$, and the normalized reproducing kernel $k_{z}^{\lambda}(w)=K_{z}^{\lambda}(w) /\left\|K_{z}^{\lambda}\right\|=K_{z}^{\lambda}(w) / \sqrt{K_{z}^{\lambda}(z)}=\prod_{i=1}^{n}((1-$ $\left.\left.\left|z_{i}\right|^{2}\right)^{\left(2+\lambda_{i}\right) / 2} /\left(1-\overline{z_{i}} w_{i}\right)^{2+\lambda_{i}}\right)$. For $z \in \mathbb{D}^{n}$, let $\phi_{z}(w)=$ $\left(\phi_{z_{1}}\left(w_{1}\right), \ldots, \phi_{z_{n}}\left(w_{n}\right)\right)$, where $\phi_{z_{i}}\left(w_{i}\right)=\left(z_{i}-w_{i}\right) /\left(1-w_{i} \overline{z_{i}}\right)$, for $i=1, \ldots, n$; then $\phi_{z}(w)$ is an automorphism on $\mathbb{D}^{n}$ that interchanges 0 and $z$. Let $\phi_{z}^{\prime}(w)=\left(\phi_{z_{1}}^{\prime}\left(w_{1}\right), \ldots, \phi_{z_{n}}^{\prime}\left(w_{n}\right)\right)$; then

$$
\left[\phi_{z}^{\prime}(w)\right]=\prod_{i=1}^{n}\left(\phi_{z_{i}}^{\prime}\left(w_{i}\right)\right)=\prod_{i=1}^{n} \frac{\left|z_{i}\right|^{2}-1}{\left(1-\overline{z_{i}} w_{i}\right)^{2}} .
$$

Given $z \in \mathbb{D}^{n}$, introduce the unitary operator $U_{z}$ on $\mathscr{A}_{\lambda}^{2}\left(\mathbb{D}^{n}\right)$ given by $U_{z} f=f \circ \phi_{z} \cdot\left[\left(\phi_{z}^{\prime}\right)^{(2+\lambda) / 2}\right]$, where $\left[\left(\phi_{z}^{\prime}\right)^{(2+\lambda) / 2}\right]=$ $\prod_{i=1}^{n}\left(\phi_{z_{i}}^{\prime}\right)^{\left(2+\lambda_{i}\right) / 2}$. It is easy to see that $U_{z}$ is self-adjoint and so $U_{z}^{2}=I$. We have $U_{0} f(w)=(-1)^{|2+\lambda| / 2} f(-w)$.

For a fixed $z \in \mathbb{D}^{n}$ we define an automorphism on the algebra $\mathscr{L}\left(\mathscr{A}_{\lambda}^{2}\right)$ of all bounded operator on $\mathscr{A}_{\lambda}^{2}\left(\mathbb{D}^{n}\right)$ by $S \mapsto$ $S_{z}:=U_{z} S U_{z} \in \mathscr{L}\left(\mathscr{A}_{\lambda}^{2}\right)$. In particular, if $S=T_{a}$ is a Toeplitz operator, then $\left(T_{a}\right)_{z}=T_{a \circ \phi_{z}}$.

The principle difference between the unit ball $\mathbb{B}^{n}$ and the polydisk $\mathbb{D}^{n}$ is that the later domain is reducible, which involves the tensor product structure of various objects introduced and studied in the paper. In particular, $L_{\lambda}^{2}\left(\mathbb{D}^{n}, d A_{\lambda}(z)\right)=L_{\lambda_{1}}^{2}\left(\mathbb{D}, d A_{\lambda_{1}}\left(z_{1}\right)\right) \otimes \cdots \otimes L_{\lambda_{n}}^{2}(\mathbb{D}$, $\left.d A_{\lambda_{n}}\left(z_{n}\right)\right)$ and $\mathscr{A}_{\lambda}^{2}\left(\mathbb{D}^{n}, d A_{\lambda}(z)\right)=\mathscr{A}_{\lambda_{1}}^{2}\left(\mathbb{D}, d A_{\lambda_{1}}\left(z_{1}\right)\right) \otimes \cdots \otimes$ $\mathscr{A}_{\lambda_{n}}^{2}\left(\mathbb{D}, d A_{\lambda_{n}}\left(z_{n}\right)\right)$. Therefore, for the orthonormal basis of $\mathscr{A}_{\lambda}^{2}\left(\mathbb{D}^{n}\right)$ and the reproducing kernel in $\mathscr{A}_{\lambda}^{2}\left(\mathbb{D}^{n}\right)$, we have $w^{\alpha}=$ $w^{\alpha_{1}} \cdots w^{\alpha_{n}}=w^{\alpha_{1}} \otimes \cdots \otimes w^{\alpha_{n}}$ and $K_{z}^{\lambda}(w)=K_{z_{1}}^{\lambda_{1}}\left(w_{1}\right) \otimes \cdots \otimes$ $K_{z_{n}}^{\lambda_{n}}\left(w_{n}\right)$.

The unitary operator $U_{z}$ on $\mathscr{A}_{\lambda}^{2}\left(\mathbb{D}^{n}\right)$ can be written by $U_{z}=U_{z_{1}} \otimes \cdots \otimes U_{z_{n}}$. In fact, $U_{z} w^{\alpha}=\phi_{z}^{\alpha}(w) \cdot\left(\phi_{z}^{\prime}(w)\right)^{(2+\lambda) / 2}=$ $\prod_{i=1}^{n}\left(\phi_{z_{i}}^{\alpha_{i}}\left(w_{i}\right)\right) \cdot\left(\phi_{z_{i}}^{\prime}\left(w_{i}\right)\right)^{\left(2+\lambda_{i}\right) / 2}=\left(\phi_{z_{1}}^{\alpha_{1}}\left(w_{1}\right)\right) \cdot\left(\phi_{z_{1}}^{\prime}\left(w_{1}\right)\right)^{\left(2+\lambda_{1}\right) / 2} \otimes$ $\cdots \otimes\left(\phi_{z_{n}}^{\alpha_{n}}\left(w_{n}\right)\right) \cdot\left(\phi_{z_{n}}^{\prime}\left(w_{n}\right)\right)^{\left(2+\lambda_{n}\right) / 2}=U_{z_{1}} w^{\alpha_{1}} \otimes \cdots \otimes U_{z_{n}} w^{\alpha_{n}}=$ $\left(U_{z_{1}} \otimes \cdots \otimes U_{z_{n}}\right)\left(w^{\alpha_{1}} \otimes \cdots \otimes w^{\alpha_{n}}\right)$. If $S \in \mathscr{L}\left(\mathscr{A}_{\lambda}^{2}\right)$ can be written by $S=S_{1} \otimes \cdots \otimes S_{n}$, then $S_{z}=S_{z_{1}} \otimes \cdots \otimes S_{z_{n}}$.

Let $S_{1}=S_{1}\left(\mathscr{A}_{\lambda}^{2}\right)$ denote the class of trace operators on $\mathscr{A}_{\lambda}^{2}\left(\mathbb{D}^{n}\right)$. Given $T \in S_{1}$, we write $\operatorname{tr}[T]$ for its trace and recall that the trace norm of $T$ is given by $\|T\|_{S_{1}}=\operatorname{tr}\left[\sqrt{T^{*} T}\right]$. Given $f, g \in \mathscr{A}_{\lambda}^{2}\left(\mathbb{D}^{n}\right)$, the rank-one-operator $f \otimes g$ acting on $\mathscr{A}_{\lambda}^{2}\left(\mathbb{D}^{n}\right)$ by the formula $(f \otimes g) h=\langle h, g\rangle_{\lambda} f$ obviously belongs to $S_{1}$. It is easily proved that $f \otimes g$ is in $S_{1}$ and with norm equal to $\|f \otimes g\|_{S_{1}}=\|f\|_{2, \lambda} \cdot\|g\|_{2, \lambda}$ and $\operatorname{tr}[f \otimes g]=\langle f, g\rangle_{\lambda}$. Recall as well that if $T \in S_{1}$ has rank $n$, then one has the inequality $\|T\|_{S_{1}} \leq \sqrt{n}\left(\operatorname{tr}\left[T^{*} T\right]\right)^{1 / 2}$. The pseudo-hyperbolic metric on $\mathbb{D}^{n}$ is defined as $\rho(z, w)=\max _{1 \leq i \leq n}\left|\phi_{z_{i}}\left(w_{i}\right)\right|$.

Throughout the paper and as a convention we will denote by $C(n, \lambda)$ a positive constant depending only on $n$ and $\lambda$ and appearing in various estimates and whose value may change at each occurrence. 


\section{The $(m, \lambda)$-Berezin Transform}

Recall that $m$-Berezin transform for unweighted Bergman space over the unit disk and over the unit polydisk was defined in [6] and [2], respectively. We will follow the recipe in [2] and first introduce some notation. Put

$$
C_{m, \alpha}=(-1)^{|\alpha|}\left(\begin{array}{c}
m \\
\alpha_{1}
\end{array}\right) \cdots\left(\begin{array}{c}
m \\
\alpha_{n}
\end{array}\right),
$$

so that, for $z, w \in \mathbb{D}^{n}$, we know

$$
\sum_{\alpha_{1}=0}^{m} \cdots \sum_{\alpha_{n}=0}^{m} C_{m, \alpha} z^{\alpha} \bar{w}^{\alpha}=\prod_{i=1}^{n}\left(1-z_{i} \overline{w_{i}}\right)^{m} .
$$

For $z \in \mathbb{D}^{n}, \lambda=\left(\lambda_{1}, \ldots, \lambda_{n}\right) \in \mathbb{R}^{n}$, and a positive integer $m$, let $K_{z}^{m+\lambda}(u)=\prod_{i=1}^{n}\left(1 /\left(1-\overline{z_{i}} u_{i}\right)^{m+\lambda_{i}+2}\right)$.

A generalization of the concept of $m$-Berezin transform to an arbitrary bounded operator on the Bergman space $\mathscr{A}^{2}\left(\mathbb{D}^{n}\right)$ requires a modification of the definition in [2].

Definition 1. We define the $(m, \lambda)$-Berezin transform of $S \in$ $\mathscr{L}\left(\mathscr{A}_{\lambda}^{2}\right)$ by

$$
\begin{aligned}
& \left(B_{m, \lambda} S\right)(z) \\
& \quad:=\frac{[\lambda+m+1]}{[\lambda+1]} \sum_{\alpha_{1}=0}^{m} \cdots \sum_{\alpha_{n}=0}^{m} C_{m, \alpha}\left\langle S_{z} w^{\alpha}, w^{\alpha}\right\rangle_{\lambda} .
\end{aligned}
$$

It is easy to see that the following pointwise estimate

$$
\begin{gathered}
\left|\left(B_{m, \lambda} S\right)(z)\right| \leq\|S\| \frac{[\lambda+m+1]}{[\lambda+1]} \sum_{\alpha_{1}=0}^{m} \cdots \sum_{\alpha_{n}=0}^{m}\left|C_{m, \alpha}\right| \\
\cdot\left\|w^{\alpha}\right\|_{\lambda}:=C(\lambda, m, n)\|S\|,
\end{gathered}
$$

where the constant $C(\lambda, m, n)>0$ is independent of $z \in \mathbb{D}^{n}$; that is, $B_{m, \lambda} S$ is a bounded function on $\mathbb{D}^{n}$ with $\left\|B_{m, \lambda} S\right\|_{\infty} \leq$ $C(\lambda, m, n)\|S\|$.

In [5], the $(m, \lambda)$-Berezin transform of $S \in \mathscr{L}\left(\mathscr{A}_{\lambda}^{2}(\mathbb{D})\right)$ is defined by $\left(B_{m, \lambda} S\right)(z)=((\lambda+m+1) /(\lambda+$ 1)) $\sum_{k=0}^{m}(-1)^{k}\left(\begin{array}{c}m \\ k\end{array}\right)\left\langle S_{z} w^{k}, w^{k}\right\rangle$, for the case of the unit disk $\mathbb{B}^{1}=\mathbb{D}$. From the point of view of the tensor product structure, given an elementary tensor $S=S_{1} \otimes \cdots \otimes S_{n} \epsilon$ $\mathscr{L}\left(\mathscr{A}_{\lambda_{1}}^{2}\left(\mathbb{D}, d A_{\lambda_{1}}\left(z_{1}\right)\right)\right) \otimes \cdots \otimes \mathscr{L}\left(\mathscr{A}_{\lambda_{n}}^{2}\left(\mathbb{D}, d A_{\lambda_{n}}\left(z_{n}\right)\right)\right)$, its $(m, \lambda)$-Berezin transform for $\lambda=\left(\lambda_{1}, \ldots, \lambda_{n}\right)$ obviously and naturally has to be defined as

$$
\begin{gathered}
\left(B_{m, \lambda} S\right)(z)=\prod_{i=1}^{n}\left(B_{m, \lambda_{i}} S_{i}\right)\left(z_{i}\right)=\prod_{i=1}^{n} \frac{\lambda_{i}+m+1}{\lambda_{i}+1} \\
\cdot \sum_{\alpha_{i}=0}^{m}(-1)^{\alpha_{i}}\left(\begin{array}{c}
m \\
\alpha_{i}
\end{array}\right)\left\langle\left(S_{i}\right)_{z_{i}} w_{i}^{\alpha_{i}}, w_{i}^{\alpha_{i}}\right\rangle_{\lambda_{i}}
\end{gathered}
$$

$$
\begin{aligned}
& =\frac{[\lambda+m+1]}{[\lambda+1]} \sum_{\alpha_{1}=0}^{m} \cdots \sum_{\alpha_{n}=0}^{m}(-1)^{|\alpha|}\left(\begin{array}{l}
m \\
\alpha_{1}
\end{array}\right) \\
& \cdots\left(\begin{array}{c}
m \\
\alpha_{n}
\end{array}\right)\left\langle\left(S_{1}\right)_{z_{1}} w_{1}^{\alpha_{1}}, w_{1}^{\alpha_{1}}\right\rangle_{\lambda_{1}} \\
& \cdots\left\langle\left(S_{n}\right)_{z_{n}} w_{n}^{\alpha_{n}}, w_{n}^{\alpha_{n}}\right\rangle_{\lambda_{n}}=\frac{[\lambda+m+1]}{[\lambda+1]} \sum_{\alpha_{1}=0}^{m} \\
& \cdots \sum_{\alpha_{n}=0}^{m} C_{m, \alpha}\left\langle S_{z} w^{\alpha}, w^{\alpha}\right\rangle_{\lambda} .
\end{aligned}
$$

Unfortunately, the set of those tensor product operators is not a linear space; that is, for any operator $S \in \mathscr{L}\left(\mathscr{A}_{\lambda}^{2}\left(\mathbb{D}^{n}\right)\right), S$ cannot be written in the form of the tensor product operators. Therefore, we define for any operator $S \in \mathscr{L}\left(\mathscr{A}_{\lambda}^{2}\left(\mathbb{D}^{n}\right)\right)$ with (10), and this coincides with Definition 1 . If $S$ can be the tensor product form, this definition is the tensor product of $\left(m, \lambda_{i}\right)$ Berezin transform for the case of $n=1$.

As usual we define the $(m, \lambda)$-Berezin transform of a function $a \in L^{\infty}\left(\mathbb{D}^{n}\right)$ by

$$
\begin{aligned}
B_{m, \lambda}(a)(z):=\left(B_{m, \lambda} T_{a}\right)(z) \\
=\frac{[\lambda+m+1]}{[\lambda+1]} \sum_{\alpha_{1}=0}^{m} \cdots \sum_{\alpha_{n}=0}^{m} C_{m, \alpha}\left\langle\left(T_{a}\right)_{z} w^{\alpha}, w^{\alpha}\right\rangle_{\lambda} .
\end{aligned}
$$

It is easy to see that $B_{m, \lambda}(a)(z)=\int_{\mathbb{D}^{n}} a \circ \phi_{z}(w) d A_{\lambda+m}(w)$. Thus, $(m, \lambda)$-Berezin transform of a Toeplitz operator $T_{a}$ acting on $\mathscr{A}_{\lambda}^{2}\left(\mathbb{D}^{n}\right)$ coincides with $(0, \lambda+m)$-Berezin transform for $T_{a}$ now considered on the weighted Bergman space $\mathscr{A}_{\lambda+m}^{2}\left(\mathbb{D}^{n}\right)$.

From the definition of $\phi_{z_{i}}\left(u_{i}\right)$, we have the identity 1 $\phi_{z_{i}}\left(u_{i}\right) \overline{\phi_{z_{i}}\left(w_{i}\right)}=\left(1-\left|z_{i}\right|^{2}\right)\left(1-\overline{w_{i}} u_{i}\right) /\left(1-\overline{z_{i}} u_{i}\right)\left(1-\overline{w_{i}} z_{i}\right)$, for $u_{i}, w_{i} \in \mathbb{D}$ and $i=1, \ldots, n$. The following proposition gives an integral representation of the $(m, \lambda)$-Berezin transform.

Proposition 2. Let $S \in \mathscr{L}\left(\mathscr{A}_{\lambda}^{2}\right), m \geqslant 0$, and $z \in \mathbb{D}^{n}$. Then

$$
\begin{gathered}
B_{m, \lambda}(S)(z)=\frac{[\lambda+m+1]}{[\lambda+1]} \prod_{i=1}^{n}\left(1-\left|z_{i}\right|^{2}\right)^{\lambda_{i}+m+2} \\
\times \int_{\mathbb{D}^{n}} \int_{\mathbb{D}^{n}}\left(1-\bar{w}_{i} u_{i}\right)^{m} K_{z}^{m+\lambda}(u) \\
\cdot \frac{K_{z}^{m+\lambda}(w) S^{*} K_{w}^{\lambda}(u)}{K^{m+\lambda}} d A_{\lambda}(u) d A_{\lambda}(w) .
\end{gathered}
$$

Proof. For $S \in \mathscr{L}\left(\mathscr{A}_{\lambda}^{2}\right)$ and $w \in \mathbb{D}^{n}$, we have $S\left(\phi_{z}^{\alpha}\right.$. $\left.\prod_{i=1}^{n}\left(\phi_{z_{i}}^{\prime}\right)^{\left(2+\lambda_{i}\right) / 2}\right)(w)=\left\langle S\left(\phi_{z}^{\alpha} \cdot \prod_{i=1}^{n}\left(\phi_{z_{i}}^{\prime}\right)^{\left(2+\lambda_{i}\right) / 2}\right), K_{w}^{\lambda}\right\rangle_{\lambda}=$ $\left\langle\phi_{z}^{\alpha} \cdot \prod_{i=1}^{n}\left(\phi_{z_{i}}^{\prime}\right)^{\left(2+\lambda_{i}\right) / 2}, S^{*} K_{w}^{\lambda}\right\rangle_{\lambda}$ and $U_{z} w^{\alpha}=\phi_{z}^{\alpha}(w)$. $\prod_{i=1}^{n}\left(\phi_{z_{i}}^{\prime}\right)^{\left(2+\lambda_{i}\right) / 2}$. Using those by (5) and (7), we have 


$$
\begin{aligned}
& B_{m, \lambda}(S)(z)=\frac{[\lambda+m+1]}{[\lambda+1]} \sum_{\alpha_{1}=0}^{m} \cdots \sum_{\alpha_{n}=0}^{m} C_{m, \alpha}\left\langle S_{z} w^{\alpha}, w^{\alpha}\right\rangle_{\lambda} \\
& =\frac{[\lambda+m+1]}{[\lambda+1]} \sum_{\alpha_{1}=0}^{m} \cdots \sum_{\alpha_{n}=0}^{m} C_{m, \alpha}\left\langle S\left(\phi_{z}^{\alpha}(w) \cdot \prod_{i=1}^{n}\left(\phi_{z_{i}}^{\prime}\right)^{\left(2+\lambda_{i}\right) / 2}\right), \phi_{z}^{\alpha}(w) \cdot \prod_{i=1}^{n}\left(\phi_{z_{i}}^{\prime}\right)^{\left(2+\lambda_{i}\right) / 2}\right\rangle_{\lambda} \\
& =\frac{[\lambda+m+1]}{[\lambda+1]} \sum_{\alpha_{1}=0}^{m} \cdots \sum_{\alpha_{n}=0}^{m} C_{m, \alpha} \times \int_{\mathbb{D}^{n}} \int_{\mathbb{D}^{n}} \phi_{z}^{\alpha}(u) \\
& \cdot \prod_{i=1}^{n}\left(\phi_{z_{i}}^{\prime}\left(u_{i}\right)\right)^{\left(2+\lambda_{i}\right) / 2} \overline{\phi_{z}^{\alpha}(w) \cdot \prod_{i=1}^{n}\left(\phi_{z_{i}}^{\prime}\left(w_{i}\right)\right)^{\left(2+\lambda_{i}\right) / 2} S^{*} K_{w}^{\lambda}(u)} d A_{\lambda}(u) d A_{\lambda}(w) \\
& =\frac{[\lambda+m+1]}{[\lambda+1]} \int_{\mathbb{D}^{n}} \int_{\mathbb{D}^{n}} \prod_{i=1}^{n}\left(1-\phi_{z_{i}}\left(u_{i}\right) \overline{\phi_{z_{i}}\left(w_{i}\right)}\right)^{m}\left(\phi_{z_{i}}^{\prime}\left(u_{i}\right)\right)^{\left(2+\lambda_{i}\right) / 2} \overline{\left(\phi_{z_{i}}^{\prime}\left(w_{i}\right)\right)^{\left(2+\lambda_{i}\right) / 2} S^{*} K_{w}^{\lambda}(u)} d A_{\lambda}(u) d A_{\lambda}(w) \\
& =\frac{[\lambda+m+1]}{[\lambda+1]} \int_{\mathbb{D}^{n}} \int_{\mathbb{D}^{n}} \prod_{i=1}^{n}\left(1-\left|z_{i}\right|^{2}\right)^{m+2+\lambda_{i}}\left(1-\overline{w_{i}} u_{i}\right)^{m} K_{z}^{m+\lambda}(u) \overline{K_{z}^{m+\lambda}(w) S^{*} K_{w}^{\lambda}(u)} d A_{\lambda}(u) d A_{\lambda}(w) \\
& =\frac{[\lambda+m+1]}{[\lambda+1]} \prod_{i=1}^{n}\left(1-\left|z_{i}\right|^{2}\right)^{\lambda_{i}+m+2} \int_{\mathbb{D}^{n}} \int_{\mathbb{D}^{n}}\left(1-\overline{w_{i}} u_{i}\right)^{m} K_{z}^{m+\lambda}(u) \overline{K_{z}^{m+\lambda}(w) S^{*} K_{w}^{\lambda}(u)} d A_{\lambda}(u) d A_{\lambda}(w) .
\end{aligned}
$$

Proposition 3. Let $S \in \mathscr{L}\left(\mathscr{A}_{\lambda}^{2}\right), m \geqslant 0$, and $z \in \mathbb{D}^{n}$. Then

$$
\begin{gathered}
B_{m, \lambda}(S)(z):=\frac{[\lambda+m+1]}{[\lambda+1]} \prod_{i=1}^{n}\left(1-\left|z_{i}\right|^{2}\right)^{\lambda_{i}+m+2} \\
\cdot \sum_{\alpha_{1}=0}^{m} \cdots \sum_{\alpha_{n}=0}^{m} C_{m, \alpha}\left\langle S\left(w^{\alpha} K_{z}^{m+\lambda}\right), w^{\alpha} K_{z}^{m+\lambda}\right\rangle_{\lambda} .
\end{gathered}
$$

Proof. We have

$$
\begin{aligned}
& \int_{\mathbb{D}^{n}} \int_{\mathbb{D}^{n}} \prod_{i=1}^{n}\left(1-\overline{w_{i}} u_{i}\right)^{m} K_{z}^{m+\lambda}(u) \\
& \cdot \overline{K_{z}^{m+\lambda}(w) S^{*} K_{w}^{\lambda}(u)} d A_{\lambda}(u) d A_{\lambda}(w) \\
& =\sum_{\alpha_{1}=0}^{m} \cdots \sum_{\alpha_{n}=0}^{m} C_{m, \alpha} \int_{\mathbb{D}^{n}} \int_{\mathbb{D}^{n}} \bar{w}^{\alpha} u^{\alpha} K_{z}^{m+\lambda}(u) \\
& \cdot \overline{K_{z}^{m+\lambda}(w) S^{*} K_{w}^{\lambda}(u)} d A_{\lambda}(u) d A_{\lambda}(w) \\
& =\sum_{\alpha_{1}=0}^{m} \cdots \sum_{\alpha_{n}=0}^{m} C_{m, \alpha} \int_{\mathbb{D}^{n}} S\left(u^{\alpha} K_{z}^{m+\lambda}\right)(w) \\
& \overline{w^{\alpha} K_{z}^{m+\lambda}(w)} d A_{\lambda}(w)=\sum_{\alpha_{1}=0}^{m} \\
& \cdots \sum_{\alpha_{n}=0}^{m} C_{m, \alpha}\left\langle S\left(w^{\alpha} K_{z}^{m+\lambda}\right), w^{\alpha} K_{z}^{m+\lambda}\right\rangle_{\lambda} .
\end{aligned}
$$

For $z, w \in \mathbb{D}^{n}$, put $t_{i}=\left(\phi_{z_{i}}\left(w_{i}\right) \overline{z_{i}}-1\right) /\left(1-z_{i} \overline{\phi_{z_{i}}\left(w_{i}\right)}\right)$, for $i=1, \ldots, n$. In ([2], P98), the map $\phi_{w_{i}} \circ \phi_{z_{i}} \circ \phi_{\phi_{w_{i}}}$ is a unitary map of $\mathbb{D}$ and maps $t_{i}$ to 1 . Let $t=\left(t_{1}, t_{2}, \ldots, t_{n}\right)$ and $t u=\left(t_{1} u_{1}, \ldots, t_{n} u_{n}\right)$, for $u \in \mathbb{D}^{n}$.

Lemma 4. For $z, w \in \mathbb{D}^{n}$,

$$
U_{w} U_{z}=V_{t} U_{\phi_{z}(w)},
$$

where $\left(V_{t} f\right)(u)=t^{(2+\lambda) / 2} f(t u)$ and $t^{(2+\lambda) / 2}=\prod_{i=1}^{n} t^{(2+\lambda) / 2}$, for $f \in \mathscr{A}_{\lambda}^{2}\left(\mathbb{D}^{n}\right)$.

Proof. Since $V_{t} w^{\alpha}=t^{(2+\lambda) / 2}(t w)^{\alpha}=t_{1}^{\left(2+\lambda_{1}\right) / 2}\left(t_{1} w_{1}\right)^{\alpha_{1}} \otimes \cdots \otimes$ $t_{n}^{\left(2+\lambda_{n}\right) / 2}\left(t_{n} w_{n}\right)^{\alpha_{n}}=V_{t_{1}} w_{1}^{\alpha_{1}} \otimes \cdots \otimes V_{t_{n}} w_{n}^{\alpha_{n}}=\left(V_{t_{1}} \otimes \cdots \otimes V_{t_{n}}\right) w^{\alpha}$, then $V_{t}=V_{t_{1}} \otimes \cdots \otimes V_{t_{n}}$. It is sufficient to show that $n=1$.

For $z, w, t \in \mathbb{D}$ and $\lambda>-1, \phi_{z} \circ \phi_{\phi_{z}(w)}(t u)=\phi_{w}(u)$. Thus for $k \geq 0$

$$
\begin{aligned}
& U_{w} U_{z} u^{k}=U_{w}\left(\phi_{z}^{k}(u) \cdot\left(\phi_{z}^{\prime}(u)\right)^{(2+\lambda) / 2}\right)=\left(\phi_{z}\right. \\
& \left.\circ \phi_{w}(u)\right)^{k} \cdot\left(\phi_{z}^{\prime} \circ \phi_{w}(u)\right)^{(2+\lambda) / 2}\left(\phi_{w}^{\prime}(u)\right)^{(2+\lambda) / 2} \\
& =\left(\phi_{\phi_{z}(w)}(t u)\right)^{k} \cdot\left(\phi_{z}^{\prime} \circ \phi_{z} \circ \phi_{\phi_{z}(w)}(t u) \cdot \phi_{z}^{\prime}\right. \\
& \left.\circ \phi_{\phi_{z}(w)}(t u) \cdot \phi_{\phi_{z}(w)}^{\prime}(t u) \cdot t\right)^{(2+\lambda) / 2}=\left(\phi_{\phi_{z}(w)}(t u)\right)^{k} \\
& \cdot\left(\phi_{\phi_{z}(w)}^{\prime}(t u) \cdot t\right)^{(2+\lambda) / 2}=V_{t} U_{\phi_{z}(w)} u^{k} .
\end{aligned}
$$

Note that $t^{(2+\lambda) / 2}$ is a complex number of modulus one.

Theorem 5. Let $S \in \mathscr{L}\left(\mathscr{A}_{\lambda}^{2}\right), m \geqslant 0$, and $z \in \mathbb{D}^{n}$; then

$$
B_{m, \lambda} S_{z}=\left(B_{m, \lambda} S\right) \circ \phi_{z} \text {. }
$$


Proof. By definition,

$$
\begin{aligned}
& \left(B_{m, \lambda} S_{z}\right)(0) \\
& =\frac{[\lambda+m+1]}{[\lambda+1]} \sum_{\alpha_{1}=0}^{m} \cdots \sum_{\alpha_{n}=0}^{m} C_{m, \alpha}\left\langle S_{z} U_{0} w^{\alpha}, U_{0} w^{\alpha}\right\rangle_{\lambda} \\
& =\frac{[\lambda+m+1]}{[\lambda+1]} \sum_{\alpha_{1}=0}^{m} \cdots \sum_{\alpha_{n}=0}^{m} C_{m, \alpha}\left\langle S_{z} w^{\alpha}, w^{\alpha}\right\rangle_{\lambda} \\
& =\left(B_{m, \lambda} S\right)(z)=\left(B_{m, \lambda} S\right) \circ \phi_{z}(0) .
\end{aligned}
$$

For any $\eta \in \mathbb{D}^{n}$, by Proposition 2, Lemma 4 , and $V_{t} V_{t}^{*}=I$, we have

$$
\begin{aligned}
& \left(B_{m, \lambda} S_{z}\right)(\eta)=\left(B_{m, \lambda} S_{z}\right) \circ \phi_{\eta}(0)=\left(B_{m, \lambda}\left(S_{z}\right)_{\eta}\right)(0) \\
& =\frac{[\lambda+m+1]}{[\lambda+1]} \int_{\mathbb{D}^{n}} \int_{\mathbb{D}^{n}} \prod_{i=1}^{n}\left(1-\overline{w_{i}} u_{i}\right)^{m} \\
& \cdot \overline{\left(\left(S_{z}\right)_{\eta}\right)^{*} K_{w}^{\lambda}(u)} d A_{\lambda}(u) d A_{\lambda}(w) \\
& =\frac{[\lambda+m+1]}{[\lambda+1]} \int_{\mathbb{D}^{n}} \int_{\mathbb{D}^{n}} \prod_{i=1}^{n}\left(1-\overline{w_{i}} u_{i}\right)^{m} \\
& \cdot \overline{U_{\eta} U_{z} S^{*} U_{z} U_{\eta} K_{w}^{\lambda}(u)} d A_{\lambda}(u) d A_{\lambda}(w) \\
& =\frac{[\lambda+m+1]}{[\lambda+1]} \int_{\mathbb{D}^{n}} \int_{\mathbb{D}^{n}} \prod_{i=1}^{n}\left(1-\overline{w_{i}} u_{i}\right)^{m} \\
& \cdot \overline{V_{t} U_{\phi_{z}(\eta)} S^{*} U_{\phi_{z}(\eta)} V_{t}^{*} K_{w}^{\lambda}(u)} d A_{\lambda}(u) d A_{\lambda}(w) \\
& =\left(B_{m, \lambda} S_{\phi_{z}(\eta)}\right)(0)=\left(B_{m, \lambda} S\right) \circ \phi_{\phi_{z}(\eta)}(0)=\left(B_{m, \lambda} S\right) \\
& \circ \phi_{z}(\eta) .
\end{aligned}
$$

Then we have $B_{m, \lambda} S_{z}=\left(B_{m, \lambda} S\right) \circ \phi_{z}$.

Lemma 6. Let $S \in \mathscr{L}\left(\mathscr{A}_{\lambda}^{2}\right)$, and $m, j \geqslant 0$. If $\left|S^{*} K_{z}^{\lambda}(w)\right| \leqslant C$ for any $w \in \mathbb{D}^{n}$, then

$$
\left(B_{m, \lambda} B_{j, \lambda}\right)(S)=\left(B_{j, \lambda} B_{m, \lambda}\right)(S) .
$$

Proof. By Theorem 5, we only prove that $\left(B_{m, \lambda} B_{j, \lambda}\right)(S)(0)=$ $\left(B_{j, \lambda} B_{m, \lambda}\right)(S)(0)$. Using Proposition 2, Fubini's theorem, and (11), we have

$$
\begin{aligned}
& B_{m, \lambda}\left(B_{j, \lambda} S\right)(0)=\frac{[\lambda+m+1]}{[\lambda+1]} \int_{\mathbb{D}^{n}} B_{j, \lambda} S \circ \phi_{0}(w) \prod_{i=1}^{n}(1 \\
& \left.-\left|w_{i}\right|^{2}\right)^{m} d A_{\lambda}(w)=\frac{[\lambda+m+1]}{[\lambda+1]} \frac{[\lambda+j+1]}{[\lambda+1]} \\
& \cdot \int_{\mathbb{D}^{n}} \prod_{i=1}^{n}\left(1-\left|w_{i}\right|^{2}\right)^{\lambda_{i}+m+j+2} \times \int_{\mathbb{D}^{n}} \int_{\mathbb{D}^{n}}\left(1-\bar{\eta}_{i} \zeta_{i}\right)^{j} K_{w}^{j+\lambda}(\zeta)
\end{aligned}
$$

$$
\begin{aligned}
& \cdot \overline{K_{w}^{j+\lambda}(\eta) S^{*} K_{\eta}^{\lambda}(\zeta)} d A_{\lambda}(\zeta) d A_{\lambda}(\eta) d A_{\lambda}(w) \\
& =\frac{[\lambda+m+1]}{[\lambda+1]} \frac{[\lambda+j+1]}{[\lambda+1]} \int_{\mathbb{D}^{n}} \int_{\mathbb{D}^{n}} F_{m, j}(\zeta, \eta) \\
& \cdot \overline{S^{*} K_{\eta}^{\lambda}(\zeta)} d A_{\lambda}(\zeta) d A_{\lambda}(\eta),
\end{aligned}
$$

where $F_{m, j}(\zeta, \eta)=\prod_{i=1}^{n}\left(1-\bar{\eta}_{i} \zeta_{i}\right)^{j} \int_{\mathbb{D}^{n}}(1 \quad-$ $\left.\left|w_{i}\right|^{2}\right)^{\lambda_{i}+m+j+2} K_{w}^{j+\lambda}(\zeta) \overline{K_{w}^{j+\lambda}(\eta)} d A_{\lambda}(w)$. Then $F_{m, j}(\zeta, \eta)=$ $\sum_{i=1}^{l} H_{i}(\zeta) \overline{G_{i}(\eta)}$, where $H_{i}$ and $G_{i}$ are holomorphic functions and for some $l \geq 0$. Then, it is sufficient to show that $F_{m, j}(\zeta, \zeta)=F_{j, m}(\zeta, \zeta)$, for $w \in \mathbb{D}^{n}$.

$$
\begin{aligned}
& F_{m, j}(\zeta, \zeta)=[\lambda+1] \prod_{i=1}^{n}\left(1-\left|\zeta_{i}\right|^{2}\right)^{j} \\
& \cdot \int_{\mathbb{D}^{n}}\left(1-\left|w_{i}\right|^{2}\right)^{2 \lambda_{i}+m+j+2}\left|K_{w}^{j+\lambda}(\zeta)\right|^{2} d A(w) \\
& =[\lambda+1] \prod_{i=1}^{n}\left(1-\left|\zeta_{i}\right|^{2}\right)^{j} \\
& \cdot \int_{\mathbb{D}^{n}}\left(1-\left|\phi_{z_{i}}\left(w_{i}\right)\right|^{2}\right)^{2 \lambda_{i}+m+j+2}\left|K_{\zeta}^{j+\lambda}\left(\phi_{z}(w)\right)\right|^{2} \\
& \cdot\left|k_{z}(w)\right|^{2} d A(w)=[\lambda+1] \prod_{i=1}^{n}\left(1-\left|\zeta_{i}\right|^{2}\right)^{m} \\
& \cdot \int_{\mathbb{D}^{n}}\left(1-\left|w_{i}\right|^{2}\right)^{2 \lambda_{i}+m+j+2}\left|K_{w}^{m+\lambda}(\zeta)\right|^{2} d A(w) \\
& =F_{j, m}(\zeta, \zeta) .
\end{aligned}
$$

Lemma 7. For any $S \in \mathscr{L}\left(\mathscr{A}_{\lambda}^{2}\right)$, there is a sequence $\left\{S_{\beta}\right\}$ satisfying $\left|S_{\beta}^{*} K_{z}^{\lambda}(w)\right| \leq C(\beta)$ for any $w \in \mathbb{D}^{n}$ and $z \in \mathbb{D}^{n}$, such that $B_{m, \lambda} S_{\beta}$ converges to $B_{m, \lambda} S$ pointwise.

Proof. Let $H^{\infty}=H^{\infty}\left(\mathbb{D}^{n}\right)$ denote the algebra of bounded holomorphic functions on $\mathbb{D}^{n}$. Both the density of $H^{\infty}$ in $\mathscr{A}_{\lambda}^{2}\left(\mathbb{D}^{n}\right)$ and the density of finite rank operator in the ideal $\mathscr{K}$ of compact operators on $\mathscr{L}\left(\mathscr{A}_{\lambda}^{2}\right)$ imply that the set $\left\{\sum_{i=1}^{l} f_{i} \otimes\right.$ $\left.g_{i}, f_{i}, g_{i} \in H^{\infty}\right\}$ is dense in the ideal $\mathscr{K}$ in the norm topology. Note that $\mathscr{K}$ is dense in the space of bounded operators on $\mathscr{A}_{\lambda}^{2}\left(\mathbb{D}^{n}\right)$ with respect to the strong operator topology. Thus, for each $S \in \mathscr{L}\left(\mathscr{A}_{\lambda}^{2}\right)$, there is a sequence $\left\{S_{\beta}\right\}$ of finite rank operators $S_{\beta}=\sum_{j=1}^{l(\beta)} f_{\beta, j} \otimes g_{\beta, j}$ converging strongly to $S$. Then Proposition 2 shows that $B_{m, \lambda} S_{\beta}$ converges to $B_{m, \lambda} S$ pointwise. To finish the proof we estimate

$$
\begin{aligned}
\left|S_{\beta}^{*} K_{z}^{\lambda}(w)\right| & =\left|\sum_{j=1}^{l(\beta)}\left(g_{\beta, j} \otimes f_{\beta, j}\right) K_{z}^{\lambda}(w)\right| \\
& =\left|\sum_{j=1}^{l(\beta)}\left\langle K_{z}^{\lambda}(w), f_{\beta, j}(w)\right\rangle_{\lambda} g_{\beta, j}(w)\right|
\end{aligned}
$$




$$
\begin{aligned}
& \leq \sum_{j=1}^{l(\beta)}\left|f_{\beta, j}(z)\right|\left|g_{\beta, j}(w)\right| \\
& \leq \sum_{j=1}^{l(\beta)}\left\|f_{\beta, j}\right\|_{\infty}\left\|g_{\beta, j}\right\|_{\infty}=C(\beta) .
\end{aligned}
$$

Proposition 8. Let $S \in \mathscr{L}\left(\mathscr{A}_{\lambda}^{2}\right)$, and $m, j \geqslant 0$; then $\left(B_{m, \lambda} B_{j, \lambda}\right)(S)=\left(B_{j, \lambda} B_{m, \lambda}\right)(S)$.

Proof. Let $S \in \mathscr{L}\left(\mathscr{A}_{\lambda}^{2}\right)$; then Lemma 7 implies that there is a sequence $\left\{S_{\alpha}\right\}$ satisfying $\left|S_{\alpha}^{*} K_{z}^{\lambda}(w)\right| \leq C(\alpha)$; hence by Lemma 6

$$
B_{m, \lambda}\left(B_{j, \lambda} S_{\alpha}\right)=B_{j, \lambda}\left(B_{m, \lambda} S_{\alpha}\right)
$$

From (11), we know that $B_{m, \lambda}\left(B_{j, \lambda} S_{\alpha}\right)=\int_{\mathbb{D}^{n}}\left(B_{j, \lambda} S_{\alpha}\right) \circ$ $\phi_{z}(w) d A_{\lambda+m}(w)$ and $\left\|\left(B_{j, \lambda} S_{\alpha}\right) \circ \phi_{z}\right\|_{\infty}=\left\|B_{j, \lambda} S_{\alpha}\right\|_{\infty} \leq$ $\left\|B_{j, \lambda}\right\| \cdot\left\|S_{\alpha}\right\| \leq C(j, \lambda)\|S\|$. Furthermore $\left(B_{j, \lambda} S_{\alpha}\right) \circ \phi_{z}(w)$ converges to $\left(B_{j, \lambda} S\right) \circ \phi_{z}(w)$. As a consequence the functions $B_{m, \lambda}\left(B_{j, \lambda} S_{\alpha}\right)$ and $B_{j, \lambda}\left(B_{m, \lambda} S_{\alpha}\right)$ converge to $\left(B_{m, \lambda} B_{j, \lambda} S\right)(z)$ and $\left(B_{j, \lambda} B_{m, \lambda} S\right)(z)$, respectively. By the uniqueness of the limit, we have $\left(B_{m, \lambda} B_{j, \lambda}\right)(S)=\left(B_{j, \lambda} B_{m, \lambda}\right)(S)$.

Theorem 9. Let $S \in \mathscr{L}\left(\mathscr{A}_{\lambda}^{2}\right)$; then there is a constant $C(n, \lambda)>$ 0 , such that

$$
\left|\left(B_{0, \lambda} S\right)(z)-\left(B_{0, \lambda} S\right)(w)\right| \leq C(n, \lambda)\|S\| \rho(z, w)
$$

Proof.

$$
\begin{aligned}
& \left|\left(B_{0, \lambda} S\right)(z)-\left(B_{0, \lambda} S\right)(w)\right|=\left|\left\langle S_{z} 1,1\right\rangle_{\lambda}-\left\langle S_{w} 1,1\right\rangle_{\lambda}\right| \\
& =\left|\operatorname{tr}\left[S_{z}(1 \otimes 1)-S_{w}(1 \otimes 1)\right]\right| \\
& =\left|\operatorname{tr}\left[S_{z}(1 \otimes 1)-S U_{z}\left(U_{z} U_{w} 1 \otimes U_{z} U_{w} 1\right) U_{z}\right]\right| \\
& =\left|\operatorname{tr}\left[S_{z}\left(1 \otimes 1-U_{\phi_{w}(z)} 1 \otimes U_{\phi_{w}(z)} 1\right)\right]\right| \\
& \leq\left\|S_{z}\right\| \cdot\left\|1 \otimes 1-U_{\phi_{w}(z)} 1 \otimes U_{\phi_{w}(z)} 1\right\|_{S_{1}} \\
& \leq \sqrt{2}\left\|S_{z}\right\|\left(2-2\left|\left\langle 1, \prod_{i=1}^{n}\left(\phi_{\phi_{w_{i}}\left(z_{i}\right)}^{\prime}\right)^{\left(2+\lambda_{i}\right) / 2}\right\rangle\right|^{2}\right)^{1 / 2} \\
& \leq 2\|S\|\left[1-\prod_{i=1}^{n}\left(1-\left|\phi_{w_{i}}\left(z_{i}\right)\right|^{2}\right)^{\left(2+\lambda_{i}\right) / 2}\right]^{1 / 2} .
\end{aligned}
$$

Let $\alpha_{i}=\phi_{w_{i}}\left(z_{i}\right)$; we have

$$
\begin{gathered}
{\left[1-\prod_{i=1}^{n}\left(1-\left|\alpha_{i}\right|^{2}\right)^{\left(2+\lambda_{i}\right) / 2}\right]} \\
\quad=1-\left(1-\left|\alpha_{1}\right|^{2}\right)^{\left(2+\lambda_{1}\right) / 2}
\end{gathered}
$$

$$
\begin{aligned}
& +\left(1-\left|\alpha_{1}\right|^{2}\right)^{\left(2+\lambda_{1}\right) / 2}\left[1-\prod_{i=2}^{n}\left(1-\left|\alpha_{i}\right|^{2}\right)^{\left(2+\lambda_{i}\right) / 2}\right] \\
\leq & C\left(\lambda_{1}\right)\left|\alpha_{1}\right|^{2}+\left[1-\prod_{i=2}^{n}\left(1-\left|\alpha_{i}\right|^{2}\right)^{\left(2+\lambda_{i}\right) / 2}\right] \ldots \\
\leq & C(n, \lambda) \max _{1 \leq i \leq n}\left|\alpha_{i}\right|^{2},
\end{aligned}
$$

where $C(n, \lambda)=n \cdot \max _{1 \leq i \leq n} C\left(\lambda_{i}\right)$; we obtain $\mid\left(B_{0, \lambda} S\right)(z)-$ $\left(B_{0, \lambda} S\right)(w) \mid \leq C(n, \lambda)\|S\| \rho(z, w)$.

Corollary 10. Let $S \in \mathscr{L}\left(\mathscr{A}_{\lambda}^{2}\right)$, and $a:=B_{0, \lambda} S \in L^{\infty}\left(\mathbb{D}^{n}\right)$; then

$$
\lim _{m \rightarrow \infty}\left\|B_{m, \lambda} a-a\right\|_{\infty}=0 .
$$

Proof. Let $\varepsilon>0$; choose $\delta>0$ with $|a(z)-a(w)|<\varepsilon$ whenever $z, w \in \mathbb{D}^{n}$ with $\rho(z, w)<\delta$. If $w \in \mathbb{D}^{n}, m \in \mathbb{N}$, by (11), we have

$$
\begin{aligned}
& \left|B_{m, \lambda}(a)(w)-a(w)\right| \leq[\lambda+m+1] \\
& \quad \cdot \int_{\mathbb{D}^{n}}\left|a \circ \phi_{w}(z)-a \circ \phi_{w}(0)\right| \\
& \quad \cdot \prod_{i=1}^{n}\left(1-\left|z_{i}\right|^{2}\right)^{\lambda_{i}+m} d A(z) \leq[\lambda+m+1] \\
& \quad \cdot\left\{\int_{\prod_{i=1}^{n} 0 \leq\left|z_{i}\right| \leq \delta}+\int_{\prod_{i=1}^{n} \delta \leq\left|z_{i}\right| \leq 1}\right\} \mid a \circ \phi_{w}(z)-a \\
& \quad \circ \phi_{w}(0) \mid \prod_{i=1}^{n}\left(1-\left|z_{i}\right|^{2}\right)^{\lambda_{i}+m} d A(z) .
\end{aligned}
$$

Denote by $I$ the first integral, and

$$
\begin{aligned}
I= & {[\lambda+m+1] \int_{\prod_{i=1}^{n} 0 \leq\left|z_{i}\right| \leq \delta}\left|B_{0, \lambda} S\left(\phi_{w}(z)-\phi_{w}(0)\right)\right| } \\
& \cdot \prod_{i=1}^{n}\left(1-\left|z_{i}\right|^{2}\right)^{\lambda_{i}+m} d A(z) \leq C(n, \lambda, \delta)\|S\| \rho(z, 0) \\
& <\varepsilon .
\end{aligned}
$$

In the first inequality we use that $\rho(\cdot, \cdot)$ is invariant under the automorphisms $\phi_{w}$ and by the Lipschitz continuity of $B_{0, \lambda} S$.

Now we estimate the second integral above.

$$
\begin{aligned}
& {[\lambda+m+1] \int_{\prod_{i=1}^{n} \delta \leq\left|z_{i}\right| \leq 1}\left|a \circ \phi_{w}(z)-a \circ \phi_{w}(0)\right|} \\
& \quad \cdot \prod_{i=1}^{n}\left(1-\left|z_{i}\right|^{2}\right)^{\lambda_{i}+m} d A(z) \leq 2[\lambda+m+1] \cdot\|a\|_{\infty} \\
& \quad \cdot \int_{\prod_{i=1}^{n} \delta \leq\left|z_{i}\right| \leq 1} \prod_{i=1}^{n}\left(1-\left|z_{i}\right|^{2}\right)^{\lambda_{i}+m} d A(z) \\
& \quad=2[\lambda+m+1]\|a\|_{\infty}\left(1-\delta^{2}\right)^{|\lambda|+m} \operatorname{vol}\left(\mathbb{D}^{n}\right) .
\end{aligned}
$$

It is clear that the right-hand side converges to zero as $m \rightarrow$ $\infty$. 


\section{Toeplitz Operators to Approximate the Bounded Operators}

In this section we will give a criterion for an operator approximated by Toeplitz operators with symbol equal to their $(m, \lambda)$-Berezin transforms. From Proposition 1.4.10 in [7] there exists Lemma 3.1 in [2].

Lemma 11 (see [2]). Suppose $a<1$ and $a+b<2$. Then

$$
\sup _{z \in \mathbb{D}^{n}} \int_{\mathbb{D}^{n}} \frac{d A(w)}{\prod_{i=1}^{n}\left(1-\left|w_{i}\right|^{2}\right)^{a}\left|1-\overline{z_{i}} w_{i}\right|^{b}}<\infty .
$$

Let $1<q<\infty$ and let $p$ be the conjugate exponent of $q$. Note that the inequality

$$
\begin{aligned}
q & =1+\frac{1}{p-1}<\max _{1 \leq i \leq n}\left\{\frac{1+2\left(\lambda_{i}+1\right)}{2+\lambda_{i}}\right\} \\
& =\max _{1 \leq i \leq n}\left\{1+\frac{1+\lambda_{i}}{2+\lambda_{i}}\right\}
\end{aligned}
$$

is equivalent to $p>\max _{1 \leq i \leq n}\left\{\left(1+2\left(\lambda_{i}+1\right)\right) /\left(1+\lambda_{i}\right)\right\}$.

Lemma 11 gives the following lemma.

Lemma 12. Let $S \in \mathscr{L}\left(\mathscr{A}_{\lambda}^{2}\right)$ and $p>\max _{1 \leq i \leq n}\left\{\left(1+2\left(\lambda_{i}+\right.\right.\right.$ 1)) $\left./\left(1+\lambda_{i}\right)\right\}$ and put $h(z)=\prod_{i=1}^{n}\left(1-\left|z_{i}\right|^{2}\right)^{-a_{i}}$ with $a_{i}=(1+$ $\left.\lambda_{i}\right)\left(2+\lambda_{i}\right) /\left(1+2\left(\lambda_{i}+1\right)\right)$, for $i=1, \ldots, n$. Then there exists $C(n, p, \lambda)$ such that

$$
\begin{gathered}
\int_{\mathbb{D}^{n}}\left|\left(S K_{z}^{\lambda}\right)(w)\right| h(w) d A_{\lambda}(w) \\
\leq C(n, p, \lambda)\left\|S_{z} 1\right\|_{p, \lambda} h(z)
\end{gathered}
$$

for all $z \in \mathbb{D}^{n}$, and

$$
\begin{aligned}
& \int_{\mathbb{D}^{n}}\left|\left(S K_{z}^{\lambda}\right)(w)\right| h(z) d A_{\lambda}(z) \\
& \quad \leq C(n, p, \lambda)\left\|S_{w}^{*} 1\right\|_{p, \lambda} h(w)
\end{aligned}
$$

for all $w \in \mathbb{D}^{n}$.

Proof. Given $z \in \mathbb{D}^{n}$, the equality $U_{z} 1=\prod_{i=1}^{n}\left(\phi_{z_{i}}^{\prime}\right)^{\left(2+\lambda_{i}\right) / 2}=$ $\prod_{i=1}^{n}(-1)^{\left(2+\lambda_{i}\right) / 2}\left(1-\left|z_{i}\right|^{2}\right)^{\left(2+\lambda_{i}\right) / 2} K_{z}^{\lambda}=(-1)^{|2+\lambda| / 2} \prod_{i=1}^{n}(1-$ $\left.\left|z_{i}\right|^{2}\right)^{\left(2+\lambda_{i}\right) / 2} K_{z}^{\lambda}$ implies that

$$
\begin{aligned}
S K_{z}^{\lambda} & =\frac{(-1)^{|2+\lambda| / 2}}{\prod_{i=1}^{n}\left(1-\left|z_{i}\right|^{2}\right)^{\left(2+\lambda_{i}\right) / 2}} S U_{z} 1 \\
& =\frac{(-1)^{|2+\lambda| / 2}}{\prod_{i=1}^{n}\left(1-\left|z_{i}\right|^{2}\right)^{\left(2+\lambda_{i}\right) / 2}}\left(S_{z} 1 \circ \phi_{z}\right) \prod_{i=1}^{n}\left(\phi_{z_{i}}^{\prime}\right)^{\left(2+\lambda_{i}\right) / 2} \\
& =S_{z} 1 \circ \phi_{z} K_{z}^{\lambda} .
\end{aligned}
$$

Thus, let $u=\phi_{z}(w)$, and apply the Hölder's inequality

$$
\begin{aligned}
& \int_{\mathbb{D}^{n}} \frac{\left|\left(S K_{z}^{\lambda}\right)(w)\right|}{\prod_{i=1}^{n}\left(1-\left|w_{i}\right|^{2}\right)^{a_{i}}} d A_{\lambda}(w)=[\lambda+1] \\
& \cdot \int_{\mathbb{D}^{n}} \frac{\left|S_{z} 1 \circ \phi_{z}(w) K_{z}^{\lambda}(w)\right|}{\prod_{i=1}^{n}\left(1-\left|w_{i}\right|^{2}\right)^{a_{i}}} \prod_{i=1}^{n}\left(1-\left|w_{i}\right|^{2}\right)^{\lambda_{i}} d A(w) \\
& =[\lambda+1] \\
& \cdot \int_{\mathbb{D}^{n}} \frac{\left|S_{z} 1(u)\right|}{\prod_{i=1}^{n}\left(1-\left|\phi_{z_{i}}\left(u_{i}\right)\right|^{2}\right)^{a_{i}-\lambda_{i}}} \prod_{i=1}^{n} \frac{\left|1-\overline{z_{i}} u_{i}\right|^{2+\lambda_{i}}}{\left(1-\left|z_{i}\right|^{2}\right)^{2+\lambda_{i}}} \\
& \cdot \frac{\left(1-\left|z_{i}\right|^{2}\right)^{2}}{\mid 1-\overline{\left.z_{i} u_{i}\right|^{4}} d A(u)=\frac{[\lambda+1]}{\prod_{i=1}^{n}\left(1-\left|z_{i}\right|^{2}\right)^{a_{i}}}} \\
& \cdot \int_{\mathbb{D}^{n}} \frac{\left|S_{z} 1(u)\right|}{\prod_{i=1}^{n}\left(1-\left|u_{i}\right|^{2}\right)^{a_{i}-\lambda_{i}}\left|1-\overline{z_{i}} u_{i}\right|^{2+\lambda_{i}-2 a_{i}}} d A(u) \\
& \quad=\frac{1}{\prod_{i=1}^{n}\left(1-\left|z_{i}\right|^{2}\right)^{a_{i}}} \\
& \cdot \int_{\mathbb{D}^{n}} \frac{\left|S_{z} 1(u)\right|}{\prod_{i=1}^{n}\left(1-\left|u_{i}\right|^{2}\right)^{a_{i}}\left|1-\overline{z_{i}} u_{i}\right|^{2+\lambda_{i}-2 a_{i}}} d A_{\lambda}(u) \\
& \quad \leq \frac{\left\|S_{z} 1\right\|}{\prod_{i=1}^{n}\left(1-\left|z_{i}\right|^{2}\right)^{a_{i}}}([\lambda+1] \\
& \left.\int_{\mathbb{D}^{n}} \frac{d A(u)}{\prod_{i=1}^{n}\left(1-\left|u_{i}\right|^{2}\right)^{a_{i} q-\lambda_{i}}\left|1-\overline{z_{i}} u_{i}\right|^{\left(2+\lambda_{i}-2 a_{i}\right) q}}\right)^{1 / q}
\end{aligned}
$$

According to (34) we have $a_{i} q-\lambda_{i}<1$ and $a_{i} q-\lambda_{i}+\left(2+\lambda_{i}-\right.$ $\left.2 a_{i}\right) q<2$, for any $i=1, \ldots, n$. Hence inequality (35) follows from Lemma 11.

The second inequality (36) follows from (35) after replacing $S$ by $S^{*}$, interchanging $w$ and $z$, and making use of

$$
\begin{aligned}
\left(S^{*} K_{w}^{\lambda}\right)(z) & =\left\langle S^{*} K_{w}^{\lambda}, K_{z}^{\lambda}\right\rangle_{\lambda}=\left\langle K_{w}^{\lambda}, S K_{z}^{\lambda}\right\rangle_{\lambda} \\
& =\overline{S K_{z}^{\lambda}(w)},
\end{aligned}
$$

which holds for all $z, w \in \mathbb{D}^{n}$.

Lemma 13. Let $S \in \mathscr{L}\left(\mathscr{A}_{\lambda}^{2}\right)$ and $p>\max _{1 \leq i \leq n}\left\{\left(1+2\left(\lambda_{i}+\right.\right.\right.$ 1)) $\left./\left(1+\lambda_{i}\right)\right\}$; then

$\|S\|$

$$
\leq C(n, p, \lambda)\left(\sup _{z \in \mathbb{D}^{n}}\left\|S_{z} 1\right\|_{p, \lambda}\right)^{1 / 2}\left(\sup _{z \in \mathbb{D}^{n}}\left\|S_{z}^{*} 1\right\|_{p, \lambda}\right)^{1 / 2},
$$

where $C(n, p, \lambda)$ is the constant of Lemma 12. 
Proof. For $f \in \mathscr{A}_{\lambda}^{2}\left(\mathbb{D}^{n}\right),(S f)(w)=\left\langle S f, K_{w}^{\lambda}\right\rangle_{\lambda}=$ $\int_{\mathbb{D}^{n}} f(z) S^{*} K_{z}^{\lambda}(w) d A_{\lambda}(z)$. Thus $S$ is the integral operator with kernel function $S^{*} K_{z}^{\lambda}(w)$. By classical Schur's Theorem [8], it is sufficient to prove that there exist positive constants $C_{1}=C(n, p, \lambda) \sup _{z \in \mathbb{D}^{n}}\left\|S_{z} 1\right\|_{p, \lambda}$ and $C_{2}=$ $C(n, p, \lambda) \sup _{z \in \mathbb{D}^{n}}\left\|S_{z}^{*} 1\right\|_{p, \lambda}$ and a positive measurable function $g$ on $\mathbb{D}^{n}$ such that $\int_{\mathbb{D}^{n}}\left|S^{*} K_{z}^{\lambda}(w)\right| g^{2}(w) d A_{\lambda}(w) \leq C_{1} g^{2}(z)$ for almost every $z \in \mathbb{D}^{n}$ and $\int_{\mathbb{D}^{n}}\left|S^{*} K_{z}^{\lambda}(w)\right| g^{2}(z) d A_{\lambda}(z) \leq$ $C_{1} g^{2}(w)$ for almost every $w \in \mathbb{D}^{n}$. By Lemma 12 , let $g=h^{1 / 2}$; we get the conclusion.

Lemma 14. Let $\left\{S_{m}\right\}$ be a bounded sequence in $\mathscr{L}\left(\mathscr{A}_{\lambda}^{2}\right)$ such that $\left\|B_{0, \lambda} S_{m}\right\|_{\infty} \rightarrow 0$ as $m \rightarrow \infty$. Then $\sup _{z \in \mathbb{D}^{n}}\left|\left\langle\left(S_{m}\right)_{z} 1, f\right\rangle\right| \rightarrow 0$ as $m \rightarrow \infty$ for any $f \in \mathscr{A}_{\lambda}^{2}\left(\mathbb{D}^{n}\right)$, and $\sup _{z \in \mathbb{D}^{n}}\left|\left(S_{m}\right)_{z} 1(\cdot)\right| \rightarrow 0$ uniformly on compact subsets of $\mathbb{D}^{n}$ as $m \rightarrow \infty$.

Proof. To prove the first statement it is sufficient to check that for each multi-index $k$

$$
\sup _{z \in \mathbb{D}^{n}}\left|\left\langle\left(S_{m}\right)_{z} 1, w^{k}\right\rangle\right| \longrightarrow 0
$$

as $m \rightarrow \infty$. Since $K_{u}^{\lambda}(w)=\prod_{i=1}^{n}\left(1 /\left(1-\overline{u_{i}} w_{i}\right)^{\lambda_{i}+2}\right)=$ $\prod_{i=1}^{n} \sum_{\alpha_{i}=0}^{\infty}\left(\Gamma\left(\alpha_{i}+\lambda_{i}+2\right) /\left(\alpha_{i}\right) ! \Gamma\left(\lambda_{i}+2\right)\right) \bar{u}_{i} \alpha_{i} w_{i}^{\alpha_{i}}=$ $\sum_{|\alpha|=0}^{\infty}(1 / \alpha !)\left[\prod_{i=1}^{n}\left(\Gamma\left(\alpha_{i}+\lambda_{i}+2\right) / \Gamma\left(\lambda_{i}+2\right)\right) \bar{u}^{\alpha} w^{\alpha}\right]$, by Proposition 3 and Theorem 5 we have

$$
\begin{aligned}
& B_{0, \lambda} S_{m}\left(\phi_{z}(u)\right)=B_{0, \lambda}\left(S_{m}\right)_{z}(u)=\prod_{i=1}^{n}\left(1-\left|u_{i}\right|^{2}\right)^{2+\lambda_{i}} \\
& \cdot\left\langle\left(S_{m}\right)_{z} K_{u}^{\lambda}, K_{u}^{\lambda}\right\rangle_{\lambda}=\prod_{i=1}^{n}\left(1-\left|u_{i}\right|^{2}\right)^{2+\lambda_{i}} \\
& \cdot \sum_{|\alpha|=0|\beta|=0}^{\infty} \sum_{\mid \beta}^{\infty} \frac{1}{\alpha !} \frac{1}{\beta !} \prod_{i=1}^{n} \frac{\Gamma\left(\alpha_{i}+\lambda_{i}+2\right)}{\Gamma\left(\lambda_{i}+2\right)} \prod_{j=1}^{n} \frac{\Gamma\left(\beta_{j}+\lambda_{j}+2\right)}{\Gamma\left(\lambda_{j}+2\right)} \\
& \cdot\left\langle\left(S_{m}\right)_{z} w^{\alpha}, w^{\beta}\right\rangle_{\lambda} \bar{u}^{\alpha} u^{\beta} .
\end{aligned}
$$

Given a multi-index $k$ and $r \in(0,1)$, we have

$$
\begin{gathered}
\int_{r \mathbb{D}^{n}} \frac{B_{0, \lambda} S_{m}\left(\phi_{z}(u)\right) \bar{u}^{k}}{\prod_{i=1}^{n}\left(1-\left|u_{i}\right|^{2}\right)^{2+\lambda_{i}}} d A_{\lambda}(u)=\sum_{|\alpha|=0}^{\infty} \sum_{|\beta|=0}^{\infty} \frac{1}{\alpha !} \frac{1}{\beta !} \\
\cdot \prod_{i=1}^{n} \frac{\Gamma\left(\alpha_{i}+\lambda_{i}+2\right)}{\Gamma\left(\lambda_{i}+2\right)} \prod_{j=1}^{n} \frac{\Gamma\left(\beta_{j}+\lambda_{j}+2\right)}{\Gamma\left(\lambda_{j}+2\right)}\left\langle\left(S_{m}\right)_{z}\right. \\
\left.\cdot w^{\alpha}, w^{\beta}\right\rangle_{\lambda} \int_{r \mathbb{D}^{n}} \bar{u}^{\alpha+k} u^{\beta} d A_{\lambda}(u)=\sum_{|\alpha|=0}^{\infty} \frac{1}{\alpha !(\alpha+k) !} \\
\cdot \prod_{i=1}^{n} \frac{\Gamma\left(\alpha_{i}+\lambda_{i}+2\right)}{\Gamma\left(\lambda_{i}+2\right)} \frac{\Gamma\left(\alpha_{i}+k_{i}+\lambda_{i}+2\right)}{\Gamma\left(\lambda_{i}+2\right)}\left\langle\left(S_{m}\right)_{z}\right.
\end{gathered}
$$

$$
\begin{aligned}
& \left.\cdot w^{\alpha}, w^{\alpha+k}\right\rangle_{\lambda} \times \int_{r \mathbb{D}^{n}}\left|u^{\alpha+k}\right|^{2} d A_{\lambda}(u) \\
& =\sum_{|\alpha|=0}^{\infty} \frac{[\lambda+1]}{\alpha !(\alpha+k) !} \\
& \cdot \prod_{i=1}^{n} \frac{\Gamma\left(\alpha_{i}+\lambda_{i}+2\right)}{\Gamma\left(\lambda_{i}+2\right)} \frac{\Gamma\left(\alpha_{i}+k_{i}+\lambda_{i}+2\right)}{\Gamma\left(\lambda_{i}+2\right)}\left\langle\left(S_{m}\right)_{z}\right. \\
& \left.\cdot w^{\alpha}, w^{\alpha+k}\right\rangle_{\lambda} \times \int_{r \mathbb{D}^{n}} \prod_{i=1}^{n}\left|u_{i}^{\alpha_{i}+\beta_{i}}\right|^{2}\left(1-\left|u_{i}\right|^{2}\right)^{\lambda_{i}} d A(u) .
\end{aligned}
$$

Passing to the polar coordinates, the integral part is $\prod_{i=1}^{n} \int_{0}^{r^{2}} \rho^{\alpha_{i}+k_{i}+1-1}(1-\rho)^{\lambda_{i}+1-1} d \rho$. Define $I_{x}(a, b)$ in the standard way ([9], Formula 8.392), $I_{x}(a, b)=(\Gamma(a+b) /$ $\Gamma(a) \Gamma(b)) \int_{0}^{x} t^{a-1}(1-t)^{b-1} d t$; then we have

$$
\begin{aligned}
& \prod_{i=1}^{n} \frac{\Gamma\left(\alpha_{i}+k_{i}+\lambda_{i}+2\right)}{\Gamma\left(\alpha_{i}+k_{i}+1\right) \Gamma\left(\lambda_{i}+1\right)} \\
& \quad \cdot \int_{0}^{r^{2}} \rho^{\alpha_{i}+k_{i}+1-1}(1-\rho)^{\lambda_{i}+1-1} d \rho \\
& \quad=\prod_{i=1}^{n} I_{r^{2}}\left(\alpha_{i}+k_{i}+1, \lambda_{i}+1\right) .
\end{aligned}
$$

In addition, (43) equals

$$
\begin{aligned}
\sum_{|\alpha|=0}^{\infty} & \frac{1}{\alpha !} \prod_{i=1}^{n} \frac{\Gamma\left(\alpha_{i}+\lambda_{i}+2\right)}{\Gamma\left(\lambda_{i}+2\right)}\left\langle\left(S_{m}\right)_{z} w^{\alpha}, w^{\alpha+k}\right\rangle_{\lambda} \\
\cdot & I_{r^{2}}\left(\alpha_{i}+k_{i}+1, \lambda_{i}+1\right)=\left\langle\left(S_{m}\right)_{z} 1, w^{k}\right\rangle_{\lambda} \\
\cdot & \prod_{i=1}^{n} I_{r^{2}}\left(k_{i}+1, \lambda_{i}+1\right)+\sum_{|\alpha|=1}^{\infty} \frac{1}{\alpha !} \\
& \cdot \prod_{i=1}^{n} \frac{\Gamma\left(\alpha_{i}+\lambda_{i}+2\right)}{\Gamma\left(\lambda_{i}+2\right)}\left\langle\left(S_{m}\right)_{z} w^{\alpha}, w^{\alpha+k}\right\rangle_{\lambda} \\
& \cdot I_{r^{2}}\left(\alpha_{i}+k_{i}+1, \lambda_{i}+1\right) .
\end{aligned}
$$

Thus we have

$$
\begin{aligned}
& \left|\left\langle\left(S_{m}\right)_{z} 1, w^{k}\right\rangle_{\lambda}\right| \\
& \quad \leq \frac{1}{\prod_{i=1}^{n} I_{r^{2}}\left(k_{i}+1, \lambda_{i}+1\right)}\left|\int_{r \mathbb{D}^{n}} \frac{B_{0, \lambda} S_{m}\left(\phi_{z}(u)\right) \bar{u}^{k}}{\prod_{i=1}^{n}\left(1-\left|u_{i}\right|^{2}\right)^{2+\lambda_{i}}} d A_{\lambda}(u)\right| \\
& \quad+\mid \sum_{|\alpha|=1}^{\infty} \frac{1}{\alpha !} \prod_{i=1}^{n} \frac{\Gamma\left(\alpha_{i}+\lambda_{i}+2\right)}{\Gamma\left(\lambda_{i}+2\right)}\left\langle\left(S_{m}\right)_{z} w^{\alpha}, w^{\alpha+k}\right\rangle_{\lambda}
\end{aligned}
$$




$$
\begin{aligned}
& \cdot \frac{I_{r^{2}}\left(\alpha_{i}+k_{i}+1, \lambda_{i}+1\right)}{I_{r^{2}}\left(k_{i}+1, \lambda_{i}+1\right)} \mid \\
& \leq \frac{[\lambda+1]\left\|B_{0, \lambda} S_{m}\right\|_{\infty}}{\prod_{i=1}^{n} I_{r^{2}}\left(k_{i}+1, \lambda_{i}+1\right)}\left|\int_{r \mathbb{D}^{n}} \frac{\left|u^{k}\right|}{\prod_{i=1}^{n}\left(1-\left|u_{i}\right|^{2}\right)^{2+\lambda_{i}}} d A(u)\right| \\
& +\sum_{|\alpha|=1}^{\infty} \frac{1}{\alpha !} \prod_{i=1}^{n} \frac{\Gamma\left(\alpha_{i}+\lambda_{i}+2\right)}{\Gamma\left(\lambda_{i}+2\right)}\left\|\left(S_{m}\right)\right\| \cdot\left\|w^{\alpha}\right\|_{2, \lambda} \cdot\left\|w^{\alpha+k}\right\|_{2, \lambda} \\
& \cdot \frac{I_{r^{2}}\left(\alpha_{i}+k_{i}+1, \lambda_{i}+1\right)}{I_{r^{2}}\left(k_{i}+1, \lambda_{i}+1\right)} \\
& \leq \frac{[\lambda+1]\left\|B_{0, \lambda} S_{m}\right\|_{\infty}}{\prod_{i=1}^{n} I_{r^{2}}\left(k_{i}+1, \lambda_{i}+1\right)}\left|\int_{r \mathbb{D}^{n}} \frac{\left|u^{k}\right|}{\prod_{i=1}^{n}\left(1-\left|u_{i}\right|^{2}\right)^{2+\lambda_{i}}} d A(u)\right| \\
& +C \sum_{|\alpha|=1}^{\infty} \prod_{i=1}^{n} \frac{I_{r^{2}}\left(\alpha_{i}+k_{i}+1, \lambda_{i}+1\right)}{I_{r^{2}}\left(k_{i}+1, \lambda_{i}+1\right)}=I+C \Sigma,
\end{aligned}
$$

where $C>0$ is a constant independent of $m$ and $z$. In the last estimate we used the boundedness of the sequence $S_{m}$ and the inequality

$$
\begin{aligned}
\frac{1}{\alpha !} & \prod_{i=1}^{n} \frac{\Gamma\left(\alpha_{i}+\lambda_{i}+2\right)}{\Gamma\left(\lambda_{i}+2\right)}\left\|w^{\alpha}\right\|_{2, \lambda} \cdot\left\|w^{\alpha+k}\right\|_{2, \lambda} \\
& =\prod_{i=1}^{n} \sqrt{\frac{\Gamma\left(\alpha_{i}+\lambda_{i}+2\right) \Gamma\left(\alpha_{i}+k_{i}+1\right)}{\left(\alpha_{i}\right) ! \Gamma\left(\alpha_{i}+k_{i}+\lambda_{i}+2\right)}} \leq 1
\end{aligned}
$$

which easily follows from (3). The first summand $I$ above tends to zero as $m \rightarrow \infty$. It is sufficient to estimate the second summand $\Sigma$.

$$
\begin{aligned}
\Sigma & =\sum_{|\alpha|=1}^{\infty} \prod_{i=1}^{n} \frac{I_{r^{2}}\left(\alpha_{i}+k_{i}+1, \lambda_{i}+1\right)}{I_{r^{2}}\left(k_{i}+1, \lambda_{i}+1\right)} \\
& =\left(\prod_{i=1}^{n} \frac{\Gamma\left(k_{i}+\lambda_{i}+2\right)}{\Gamma\left(k_{i}+1\right) \Gamma\left(\lambda_{i}+1\right)}\right. \\
& \left.\cdot \int_{0}^{r^{2}} \rho^{k_{i}}(1-\rho)^{\lambda_{i}} d \rho\right)^{-1} \\
& \times \sum_{|\alpha|=1}^{\infty} \prod_{i=1}^{n} \frac{\Gamma\left(\alpha_{i}+k_{i}+\lambda_{i}+2\right)}{\Gamma\left(\alpha_{i}+k_{i}+1\right) \Gamma\left(\lambda_{i}+1\right)} \\
& \cdot \int_{0}^{r^{2}} \rho^{\alpha_{i}+k_{i}}(1-\rho)^{\lambda_{i}} d \rho .
\end{aligned}
$$

Estimating the multiple $(1-\rho)^{\lambda_{i}}$ for any $i=1, \ldots, n$ in both integrals $\left(1-r^{2}\right)^{\lambda_{i}} \leq(1-\rho)^{\lambda_{i}} \leq 1$, for $\lambda_{i} \geq 0$, and $1 \leq$ $(1-\rho)^{\lambda_{i}} \leq\left(1-r^{2}\right)^{\lambda_{i}}$, for $\lambda_{i} \in(-1,0)$. By ([9], Formula 8.328.2),

$$
\lim _{\alpha_{i} \rightarrow \infty} \frac{\Gamma\left(\alpha_{i}+k_{i}+\lambda_{i}+2\right)}{\Gamma\left(\alpha_{i}+k_{i}+1\right)\left(\alpha_{i}+k_{i}+1\right)^{\lambda_{i}+1}}=1,
$$

and thus there exists $C_{i}>0$ such that, for all $\alpha \in \mathbb{Z}_{+}^{n}, \Gamma\left(\alpha_{i}+\right.$ $\left.k_{i}+\lambda_{i}+2\right) / \Gamma\left(\alpha_{i}+k_{i}+1\right)\left(\alpha_{i}+k_{i}+1\right)^{\lambda_{i}+1}<C_{i}$. Then

$$
\begin{aligned}
\Sigma \leq & \prod_{i=1}^{n} \frac{\Gamma\left(k_{i}+1\right) \Gamma\left(\lambda_{i}+1\right)\left(k_{i}+1\right)}{\Gamma\left(k_{i}+\lambda_{i}+2\right)} \frac{\left(1-r^{2}\right)^{-\left|\lambda_{i}\right|}}{r^{2 k_{i}+2}} \\
& \cdot \sum_{|\alpha|=1}^{\infty} \prod_{i=1}^{n} \frac{\Gamma\left(\alpha_{i}+k_{i}+\lambda_{i}+2\right)}{\Gamma\left(\alpha_{i}+k_{i}+1\right) \Gamma\left(\lambda_{i}+1\right)} \frac{r^{2\left(\alpha_{i}+k_{i}\right)+2}}{\left(\alpha_{i}+k_{i}+1\right)} \\
& \leq \prod_{i=1}^{n} C_{i} \frac{\Gamma\left(k_{i}+2\right)}{\Gamma\left(k_{i}+\lambda_{i}+2\right)}\left(1-r^{2}\right)^{-\left|\lambda_{i}\right|} \\
& \cdot \sum_{|\alpha|=1}^{\infty} \prod_{i=1}^{n}\left(\alpha_{i}+k_{i}+1\right)^{\lambda_{i}} r^{2 \alpha_{i}} .
\end{aligned}
$$

The power series in $r$ in the last line has radius of convergence equal to 1 and vanishes at 0 . Thus the value of $\Sigma$ becomes small if one takes $r$ sufficiently closed to 0 .

In order to prove the second statement we use the series representations (3) and (4) again,

$$
\begin{aligned}
& \left|\left(S_{m}\right)_{z} 1(u)\right|=\left|\left\langle\left(S_{m}\right)_{z} 1, K_{u}^{\lambda}\right\rangle_{\lambda}\right| \\
& \leq\left|\left\langle\left(S_{m}\right)_{z} 1, \sum_{|\alpha|=0}^{\infty} \frac{1}{\alpha !}\left[\prod_{i=1}^{n} \frac{\Gamma\left(\alpha_{i}+\lambda_{i}+2\right)}{\Gamma\left(\lambda_{i}+2\right)} \bar{u}^{\alpha} w^{\alpha}\right]\right\rangle_{\lambda}\right| \\
& \leq \sum_{|\alpha|=0}^{\infty} \frac{1}{\alpha !} \prod_{i=1}^{n} \frac{\Gamma\left(\alpha_{i}+\lambda_{i}+2\right)}{\Gamma\left(\lambda_{i}+2\right)}\left|\left\langle\left(S_{m}\right)_{z} 1, w^{\alpha}\right\rangle_{\lambda}\right| \cdot\left|u^{\alpha}\right| \\
& \leq \sum_{|\alpha|=0}^{l-1} \frac{1}{\alpha !} \prod_{i=1}^{n} \frac{\Gamma\left(\alpha_{i}+\lambda_{i}+2\right)}{\Gamma\left(\lambda_{i}+2\right)}\left|\left\langle\left(S_{m}\right)_{z} 1, w^{\alpha}\right\rangle_{\lambda}\right| \\
& \quad+\sum_{|\alpha|=l}^{\infty} \frac{1}{\alpha !} \prod_{i=1}^{n} \frac{\Gamma\left(\alpha_{i}+\lambda_{i}+2\right)}{\Gamma\left(\lambda_{i}+2\right)}\left|\left\langle\left(S_{m}\right)_{z} 1, w^{\alpha}\right\rangle_{\lambda}\right| \cdot\left|u^{\alpha}\right| \\
& \leq \sum_{|\alpha|=0}^{l-1} \frac{1}{\alpha !} \prod_{i=1}^{n} \frac{\Gamma\left(\alpha_{i}+\lambda_{i}+2\right)}{\Gamma\left(\lambda_{i}+2\right)}\left|\left\langle\left(S_{m}\right)_{z} 1, w^{\alpha}\right\rangle_{\lambda}\right| \\
& \quad+\sum_{|\alpha|=l}^{\infty} \frac{1}{\alpha !} \prod_{i=1}^{n} \frac{\Gamma\left(\alpha_{i}+\lambda_{i}+2\right)}{\Gamma\left(\lambda_{i}+2\right)}\left\|S_{m}\right\| \cdot\left\|w^{\alpha}\right\|_{\lambda} \cdot\left|u^{\alpha}\right| \\
& \leq \sum_{|\alpha|=0}^{l-1} \frac{1}{\alpha !} \prod_{i=1}^{n} \frac{\Gamma\left(\alpha_{i}+\lambda_{i}+2\right)}{\Gamma\left(\lambda_{i}+2\right)}\left|\left\langle\left(S_{m}\right)_{z} 1, w^{\alpha}\right\rangle_{\lambda}\right| \\
& \quad+C \sum_{|\alpha|=l}^{\infty}\left(\prod_{i=1}^{n} \frac{\Gamma\left(\alpha_{i}+\lambda_{i}+2\right)}{\left(\alpha_{i}\right) ! \Gamma\left(\lambda_{i}+2\right)}\right)^{1 / 2}\left|u^{\alpha}\right|=\Sigma_{1}+\Sigma_{2} .
\end{aligned}
$$

By the first statement of the lemma the expression $\Sigma_{1}$ uniformly tends to zero as $m \rightarrow \infty$ with $l$ being already 
fixed. To estimate $\Sigma_{2}$ we use the Cauchy-Schwarz inequality,

$$
\begin{aligned}
\Sigma_{2} & =C \sum_{j=l}^{\infty} \sum_{|\alpha|=j}\left(\prod_{i=1}^{n} \frac{\Gamma\left(\alpha_{i}+\lambda_{i}+2\right)}{\left(\alpha_{i}\right) ! \Gamma\left(\lambda_{i}+2\right)}\right)^{1 / 2}\left|u^{\alpha}\right| \\
& \leq C \sum_{j=l}^{\infty}\left(\frac{1}{j !}\right)^{1 / 2} \prod_{i=1}^{n}\left(\frac{\Gamma\left(j+\lambda_{i}+2\right)}{\Gamma\left(\lambda_{i}+2\right)}\right)^{1 / 2} \\
& \cdot \sum_{|\alpha|=j}\left[\frac{j !}{\alpha !}\right]^{1 / 2}\left|u^{\alpha}\right| \leq C \sum_{j=l}^{\infty}\left(\frac{1}{j !}\right)^{1 / 2} \\
& \cdot \prod_{i=1}^{n}\left(\frac{\Gamma\left(j+\lambda_{i}+2\right)}{\Gamma\left(\lambda_{i}+2\right)}\right)^{1 / 2}\left(\sum_{|\alpha|=j} \frac{j !}{\alpha !}\left|u^{\alpha}\right|^{2}\right)^{1 / 2} \\
& \cdot\left(\sum_{|\alpha|=j} 1\right)^{1 / 2}=C \sum_{j=l}^{\infty}\left(\frac{1}{j !}\right)^{1 / 2} \\
& \cdot \prod_{i=1}^{n}\left(\frac{\Gamma\left(j+\lambda_{i}+2\right)}{\Gamma\left(\lambda_{i}+2\right)}\right)^{1 / 2}\left(\sum_{|\alpha|=j} \frac{j !}{\alpha !}\left|u^{\alpha}\right|^{2}\right)^{1 / 2} \\
& \cdot\left(\frac{(n+j-1) !}{j !(n-1) !}\right)^{1 / 2} \cdot
\end{aligned}
$$

In [5], we get $\sum_{|\alpha|=j}(j ! / \alpha !)\left|u^{\alpha}\right|^{2}=|u|^{2 j}$; we finally have $\Sigma_{2} \leq$ $C \sum_{j=l}^{\infty}(1 / j !)^{1 / 2}((n+j-1) ! / j !(n-1) !)^{1 / 2} \prod_{i=1}^{n}\left(\Gamma\left(j+\lambda_{i}+2\right) / \Gamma\left(\lambda_{i}+\right.\right.$ 2) $)^{1 / 2} r^{j}$. By choosing $l$ sufficiently large we can make $\Sigma_{2}$ as small as needed. This ends the proof.

Lemma 15. Let $\left\{S_{m}\right\}$ be a bounded sequence in $\mathscr{L}\left(\mathscr{A}_{\lambda}^{2}\right)$ such that $\left\|B_{0, \lambda} S_{m}\right\|_{\infty} \rightarrow 0$ as $m \rightarrow \infty$. Assume that, for some $p>$ $\max _{1 \leq i \leq n}\left\{\left(1+2\left(\lambda_{i}+1\right)\right) /\left(1+\lambda_{i}\right)\right\}$, the following inequalities hold:

$$
\begin{aligned}
& \sup _{z \in \mathbb{D}^{n}}\left\|\left(S_{m}\right)_{z} 1\right\|_{p, \lambda} \leq C, \\
& \sup _{z \in \mathbb{D}^{n}}\left\|\left(S_{m}^{*}\right)_{z} 1\right\|_{p, \lambda} \leq C,
\end{aligned}
$$

where $C>0$ is independent of $m$. Then $S_{m} \rightarrow 0$ as $m \rightarrow \infty$ in the $\mathscr{L}\left(\mathscr{A}_{\lambda}^{2}\right)$-norm.

Proof. By Lemma 13,

$$
\begin{array}{r}
\left\|S_{m}\right\| \leq C(n, p, \lambda)\left(\sup _{z \in \mathbb{D}^{n}}\left\|\left(S_{m}\right)_{z} 1\right\|_{p, \lambda}\right)^{1 / 2} \\
\cdot\left(\sup _{z \in \mathbb{D}^{n}}\left\|\left(S_{m}^{*}\right)_{z} 1\right\|_{p, \lambda}\right)^{1 / 2} \leq C(n, p, \lambda) .
\end{array}
$$

Then, for $\max _{1 \leq i \leq n}\left\{\left(1+2\left(\lambda_{i}+1\right)\right) /\left(1+\lambda_{i}\right)\right\}<s<p$, Hölder's inequality gives

$$
\begin{aligned}
& \sup _{z \in \mathbb{D}^{n}}\left\|\left(S_{m}\right)_{z} 1\right\|_{s, \lambda}^{s} \leq \sup _{z \in \mathbb{D}^{n}} \int_{\mathbb{D}^{n} \mid r \overline{\mathbb{D}^{n}}}\left|\left(S_{m}\right)_{z} 1(w)\right|^{s} d A_{\lambda}(w) \\
& \quad+\sup _{z \in \mathbb{D}^{n}} \int_{r \overline{\mathbb{D}^{n}}}\left|\left(S_{m}\right)_{z} 1(w)\right|^{s} d A_{\lambda}(w) \\
& \quad \leq\left(\sup _{z \in \mathbb{D}^{n}} \int_{\mathbb{D}^{n} r \overline{\mathbb{D}^{n}}}\left(\left|\left(S_{m}\right)_{z} 1(w)\right|^{s}\right)^{p / s} d A_{\lambda}(w)\right)^{s / p} \\
& \quad \cdot\left(\int_{\mathbb{D}^{n} \mid r \overline{\mathbb{D}^{n}}} d A_{\lambda}(w)\right)^{(1-s / p)} \\
& \quad+\sup _{z \in \mathbb{D}^{n}} \int_{r \mathbb{\mathbb { D }}^{n}}\left|\left(S_{m}\right)_{z} 1(w)\right|^{s} d A_{\lambda}(w) \\
& \quad \leq \sup _{z \in \mathbb{D}^{n}}\left\|\left(S_{m}\right)_{z} 1\right\|_{p, \lambda}^{s}\left(\int_{\mathbb{D}^{n} \mid r \overline{\mathbb{D}^{n}}} d A_{\lambda}(w)\right)^{(1-s / p)} \\
& \quad+\sup _{z \in \mathbb{D}^{n}} \int_{r \overline{\mathbb{D}^{n}}}\left|\left(S_{m}\right)_{z} 1(w)\right|^{s} d A_{\lambda}(w) .
\end{aligned}
$$

Then the first term is less than or equal to $C^{s}\left(n\left(1-r^{2}\right)^{\lambda+1} /(\lambda+\right.$ $1))^{(1-s / p)}$ which converges to 0 as $r$ goes to 1 and the second term tends to 0 as $m \rightarrow \infty$ by Lemma 14. Finally, Lemma 13 gives

$$
\begin{aligned}
& \left\|S_{m}\right\| \leq C(n, s, \lambda)\left(\sup _{z \in \mathbb{D}^{n}}\left\|\left(S_{m}\right)_{z} 1\right\|_{s, \lambda}\right)^{1 / 2} \\
& \cdot\left(\sup _{z \in \mathbb{D}^{n}}\left\|\left(S_{m}^{*}\right)_{z} 1\right\|_{s, \lambda}\right)^{1 / 2} \leq C(n, s, \lambda) \\
& \cdot\left(\sup _{z \in \mathbb{D}^{n}}\left\|\left(S_{m}\right)_{z} 1\right\|_{s, \lambda}\right)^{1 / 2}\left(\sup _{z \in \mathbb{D}^{n}}\left\|\left(S_{m}^{*}\right)_{z} 1\right\|_{p, \lambda}\right)^{1 / 2} \\
& \longrightarrow 0,
\end{aligned}
$$$$
\text { as } m \rightarrow 0 \text {, proving the statement of the lemma. }
$$

Corollary 16. Let $S \in \mathscr{L}\left(\mathscr{A}_{\lambda}^{2}\right)$ such that, for some $p>$ $\max _{1 \leq i \leq n}\left\{\left(1+2\left(\lambda_{i}+1\right)\right) /\left(1+\lambda_{i}\right)\right\}$, the following inequalities hold

$$
\begin{gathered}
\sup _{z \in \mathbb{D}^{n}}\left\|S_{z} 1-\left(T_{B_{m, \lambda} S}\right)_{z} 1\right\|_{p, \lambda} \leq C, \\
\sup _{z \in \mathbb{Q}^{n}}\left\|S_{z}^{*} 1-\left(T_{B_{m, \lambda}} S^{*}\right)_{z} 1\right\|_{p, \lambda} \leq C,
\end{gathered}
$$

where $C>0$ is independent of $m$. Then $T_{B_{m, \lambda} S} \rightarrow S$ as $m \rightarrow \infty$ in the $\mathscr{L}\left(\mathscr{A}_{\lambda}^{2}\right)$-norm.

Proof. Let $S_{m}=S-T_{B_{m, \lambda} S}$ and by Proposition 8, we have

$$
\begin{aligned}
B_{0, \lambda} S_{m} & =B_{0, \lambda} S-B_{0, \lambda} T_{B_{m, \lambda} S}=B_{0, \lambda} S-B_{0, \lambda}\left(B_{m, \lambda} S\right) \\
& =B_{0, \lambda} S-B_{m, \lambda}\left(B_{0, \lambda} S\right) .
\end{aligned}
$$


By Corollary 10, the right of equation uniformly tends to 0 as $m \rightarrow \infty$; that is, $\left\|B_{0, \lambda} S_{m}\right\|_{\infty} \rightarrow 0$. An application of Lemma 15 finishes the proof.

Theorem 17. Let $S \in \mathscr{L}\left(\mathscr{A}_{\lambda}^{2}\right)$. If there is $p>\max _{1 \leq i \leq n}\{(1+$ $\left.\left.2\left(\lambda_{i}+1\right)\right) /\left(1+\lambda_{i}\right)\right\}$, such that

$$
\begin{aligned}
& \sup _{z \in \mathbb{D}^{n}}\left\|T_{\left(B_{m, \lambda} S\right) \circ \phi_{z}} 1\right\|_{p, \lambda} \leq C, \\
& \sup _{z \in \mathbb{D}^{n}}\left\|T_{\left(B_{m, \lambda} S\right) \circ \phi_{z}}^{*} 1\right\|_{p, \lambda} \leq C,
\end{aligned}
$$

where $C>0$ is independent of $m$, then $T_{B_{m, \lambda} S} \rightarrow S$ as $m \rightarrow \infty$ in the $\mathscr{L}\left(\mathscr{A}_{\lambda}^{2}\right)$-norm.

Proof. Since $T_{\left(B_{m, \lambda} S\right) \circ \phi_{z}}=\left(T_{B_{m, \lambda} S}\right)_{z}$ and $T_{\left(B_{m, \lambda} S\right) \circ \phi_{z}}^{*}=T_{B_{m, \lambda} S_{z}}^{*}=$ $T_{\overline{B_{m, \lambda} S_{z}}}=T_{B_{m, \lambda} S_{z}^{*}}=T_{\left(B_{m, \lambda} S^{*}\right) \circ \phi_{z}}$, by Corollary 16, we only need to prove that (59) implies (53). Hence, it is sufficient to prove that

$$
\sup _{z \in \mathbb{D}^{n}}\left\|S_{z} 1\right\|_{p, \lambda}<\infty .
$$

By Lemma 13, we have

$$
\begin{gathered}
\left\|T_{B_{m, \lambda}} S\right\| \leq C(n, p, \lambda)\left(\sup _{z \in \mathbb{D}^{n}}\left\|T_{\left(B_{m, \lambda} S\right) \circ \phi_{z}} 1\right\|_{p, \lambda}\right)^{1 / 2} \\
\cdot\left(\sup _{z \in \mathbb{D}^{n}}\left\|T_{\left(B_{m, \lambda} S\right)_{\circ} \phi_{z}}^{*} 1\right\|_{p, \lambda}\right)^{1 / 2}<C,
\end{gathered}
$$

where $C$ is independent of $m$. Let $S_{m}=S-T_{B_{m, \lambda} S}$; then $\left\|S_{m}\right\| \leq C$, where $C$ is independent of $m$. According to the proof of Corollary 16, we get $\lim _{m \rightarrow \infty}\left\|B_{0, \lambda} S_{m}\right\|_{\infty}=0$. Let $f$ be an analytic polynomial with $\|f\|_{q, \lambda}=1$; Lemma 14 implies $\sup _{z \in \mathbb{D}^{n}}\left|\left\langle\left(S_{m}\right)_{z} 1, f\right\rangle\right| \rightarrow 0$ as $m \rightarrow \infty$. Then, for any $\varepsilon>0$ and any $z_{0} \in \mathbb{D}^{n}$, there is a sufficiently large $m$ such that

$$
\begin{aligned}
\left|\left\langle S_{z_{0}} 1, f\right\rangle_{\lambda}\right| \leq & \left|\left\langle\left(S_{m}\right)_{z_{0}} 1, f\right\rangle_{\lambda}\right|+\left|\left\langle\left(T_{B_{m, \lambda} S}\right)_{z_{0}} 1, f\right\rangle_{\lambda}\right| \\
\leq & \sup _{z \in \mathbb{D}^{n}}\left|\left\langle\left(S_{m}\right)_{z} 1, f\right\rangle_{\lambda}\right| \\
& +\left|\left\langle\left(T_{B_{m, \lambda} S}\right)_{z_{0}} 1, f\right\rangle_{\lambda}\right| \leq \varepsilon+C,
\end{aligned}
$$

where $C$ is independent of $m$ and $z_{0}$. Since $\varepsilon$ is arbitrary, we have inequality (60).

\section{Competing Interests}

The authors declare that they have no competing interests.

\section{Acknowledgments}

This research is supported by NSFC, Item no. 11271059.

\section{References}

[1] M. Engliš, "Density of algebras generated by Toeplitz operators on Bergman spaces," Arkiv för Matematik, vol. 30, no. 2, pp. 227243, 1992.
[2] K. Nam and D. Zheng, " $m$-Berezin transform on the polydisk," Integral Equations and Operator Theory, vol. 56, no. 1, pp. 93-113, 2006.

[3] K. Nam, D. Zheng, and C. Zhong, " $m$-Berezin transform and compact operators," Revista Matemática Iberoamericana, vol. 22, no. 3, pp. 867-892, 2006.

[4] M. Mitkovski, D. Suárez, and B. D. Wick, “The essential norm of operators on $\mathscr{A}_{\alpha}^{P}\left(\mathbb{B}_{n}\right)$," Integral Equations and Operator Theory, vol. 75, no. 2, pp. 197-233, 2013.

[5] W. Bauer, C. H. Yañez, and N. Vasilevski, “ $(m, \lambda)$-Berezin transform and approximation of operators on weighted bergman spaces over the unit ball," Operator Theory: Advances and Applications, vol. 240, pp. 45-68, 2014.

[6] D. Suárez, "Approximation and symbolic calculus for Toeplitz algebras on the Bergman space," Revista Matemática Iberoamericana, vol. 20, no. 2, pp. 563-610, 2004.

[7] W. Rudin, Function Theory in the Unit Ball of $C^{n}$, Springer, Berlin, Germany, 1980.

[8] K. Zhu, Operator Theory in Function Spaces, Marcel Dekker Inc, 1990.

[9] I. S. Gradshteyn and I. M. Ryzhik, Tables if Integrals, Series, and Products, Academic Press, New York, NY, USA, 1980. 


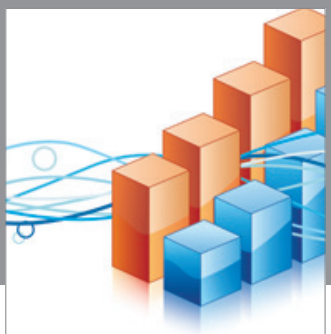

Advances in

Operations Research

vatem alat4

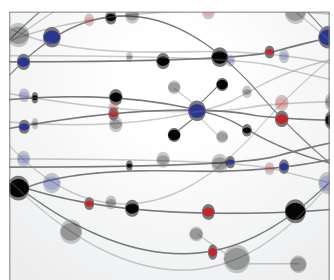

\section{The Scientific} World Journal
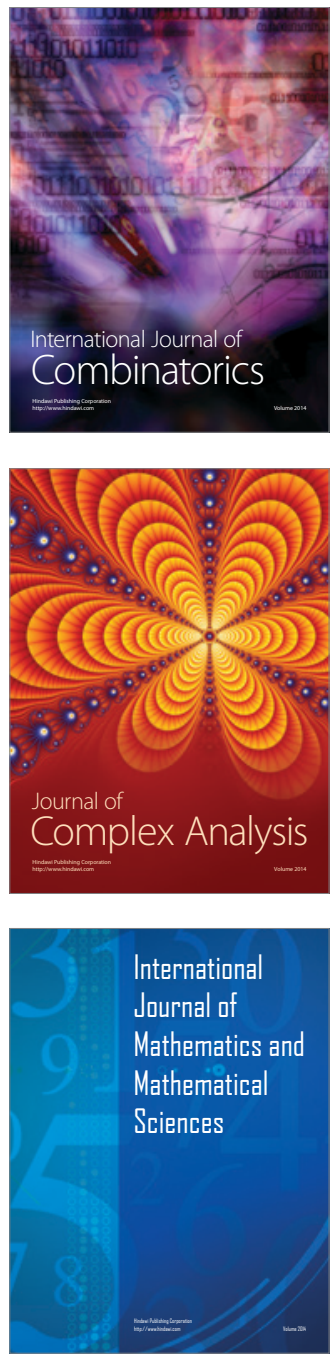
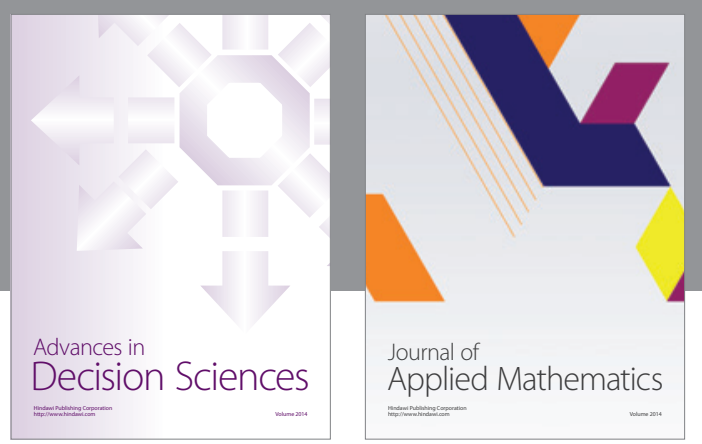

Algebra

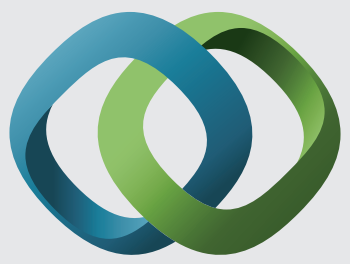

\section{Hindawi}

Submit your manuscripts at

http://www.hindawi.com
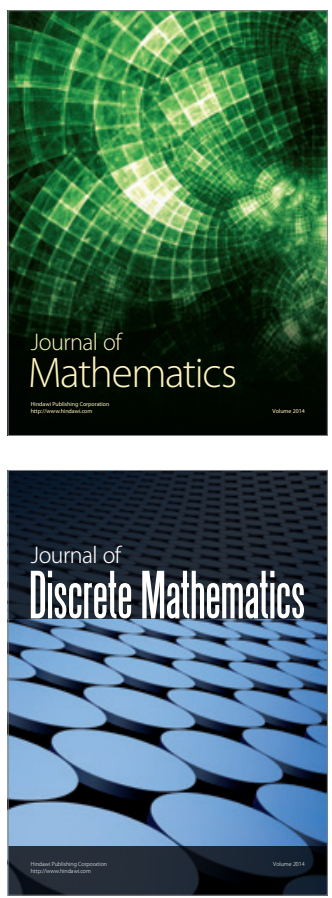

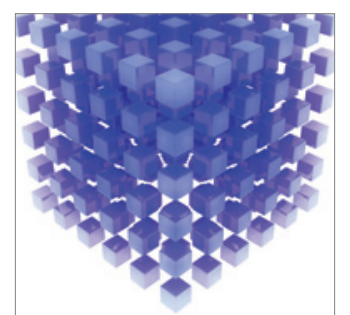

Mathematical Problems in Engineering
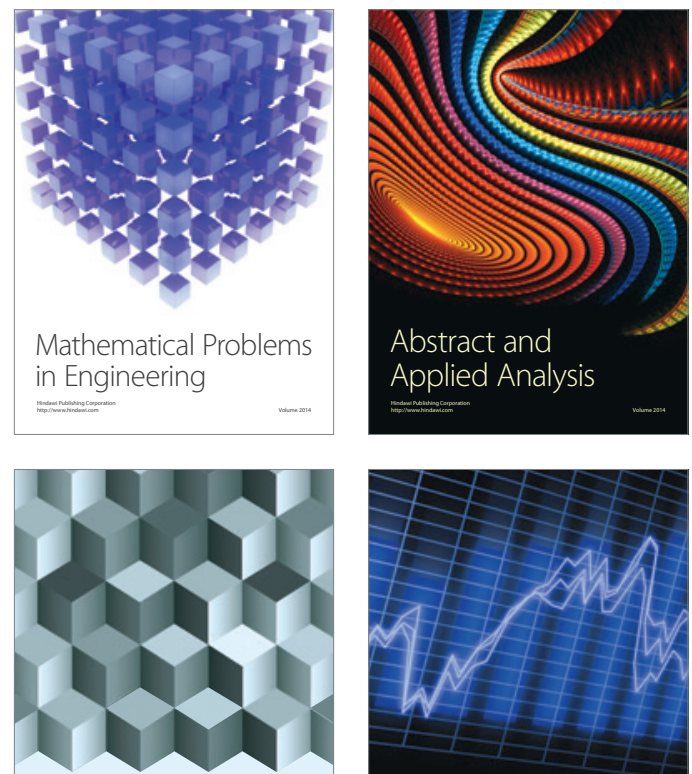

Journal of

Function Spaces

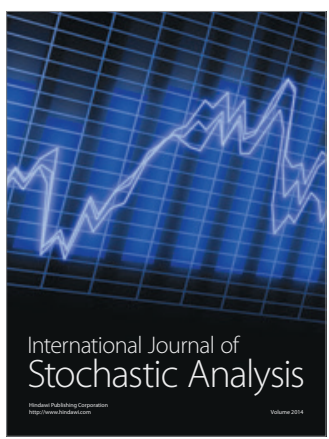

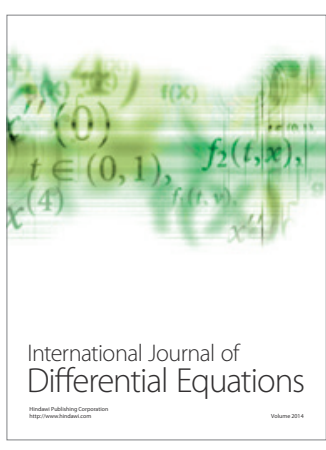
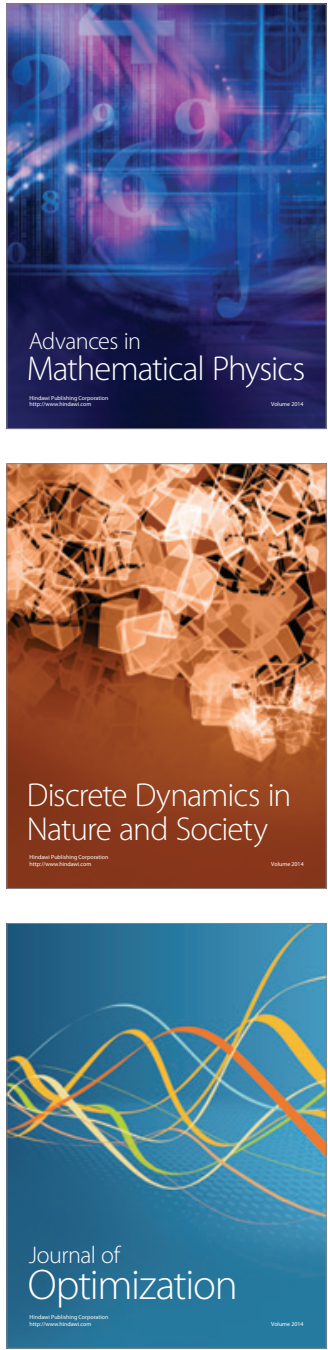